\title{
Renormalization of Supersymmetric Field Theories in Loop Regularization with String-mode Regulators
}

\author{
Jian-Wei Cui, Yong Tang and Yue-Liang Wu \\ Kavli Institute for Theoretical Physics China, \\ Key Laboratory of Frontiers in Theoretical Physics, Institute of Theoretical Physics, \\ Chinese Academy of Sciences, Beijing 100190, China
}

\begin{abstract}
By applying the recently developed Loop Regularization(LR) with string-mode regulators to supersymmetric field theories, we explicitly verify the supersymmetric Ward identities in several supersymmetric models at one-loop level. It is interesting to observe that supersymmetry is a so remarkable symmetry that the supersymmetric Ward identities hold as long as a regularization scheme is realized in the exact four dimensional space-time with translational invariance for the momentum integration, and the gauge symmetry can be maintained once the regularization scheme preserves supersymmetry and satisfies the consistency condition for logarithmic divergences. As a manifest demonstration, we carry out a complete one-loop renormalization for the massive Wess-Zumino model by adopting the LR method, it is found that all the quadratic divergences cancel out and the relations among masses and coupling constants hold after renormalization, which agrees with the well-known non-renormalization theorem. It is concluded that the LR method preserves not only gauge symmetry but also supersymmetry. A simple and definite derivation of Majorana Feynman rules is found to be very useful.
\end{abstract}

PACS numbers: 11.10.-z,11.15.-q,11.10.Gh,11.30.Pb 


\section{INTRODUCTION}

Supersymmetry has attracted physicists for several decades since it was proposed in 1970s[1]. As it is well-known that symmetry has played an important role in particle physics, and three of the four basic forces in nature are governed by gauge symmetries and have successfully been described by quantum field theory. While quantum field theories are bothered by the infinities which must be regularized to be well-defined. On the other hand, whether the symmetries of classical Lagrangian still hold in the quantum level remains an important issue, this is because some times it is difficult to distinguish between a real anomaly and an apparent violation of the symmetries due to the use of a symmetry-violating regularization method. In general, when a symmetry of original Lagrangian is still a symmetry of a full quantum effective action, such a symmetry is regarded to be preserved in the quantum level, but there are several exceptions such as chiral anomaly. Thus one may ask whether supersymmetry is a symmetry of the full quantum theory. This question has been studied in a regularization-independent way in ref.[2], and the answer is yes. This means when investigating the quantum effects of the supersymmetric theories, one must adopt a supersymmetry-preserving regularization method.

Several regularization methods have been applied to supersymmetric theories, such as dimensional reduction(DRED) [3], differential regularization [4] and the so-called implicit regularization [5], among them DRED is the most common one. It has been shown that DRED can preserve supersymmetry in several models [6, 7, 8]. Strictly speaking, DRED is mathematically inconsistent [9, 10] to be applied to the supersymmetric theories, which is similar to the case when it is applied to the chiral theories, this is because both supersymmetry and the definition of $\gamma_{5}$ require an exact dimension. A consistent regularization method that can be applied to all possible cases in quantum field theories is needed. In this sense, the recently developed loop regularization(LR) with stringmode regulators [11, 12] may deserve a special attention, it has successfully been applied to the calculations of triangle anomaly of QED with clarifying the possible ambiguities caused by $\gamma_{5}[13]$, the evaluation of a consistent coefficient of the CPT and Lorentz symmetry breaking Chern-Simons term[14], the computation of all the one-loop renormalization constants for the non-Abelian gauge theory and the determination for the coefficient of QCD $\beta$ function[15], and the derivation of the chiral effective field theory with a dynamically generated spontaneous symmetry breaking [16]. The key concept of this new regularization method is the introduction of the irreducible loop integrals(ILIs) which are evaluated from Feynman integrals by using Feynman parameter method.

It has been shown that the LR method can preserve the non-Abelian gauge symmetry, and meanwhile maintain the divergent behavior of original field theories. In particular, the LR method is realized in the original four dimensional space-time with translational and Lorentz invariance even if two intrinsic mass scales are introduced, thus it can balance the bosonic and fermionic degrees automatically and there is also no ambiguity about the definition of $\gamma_{5}$. It is then believed that this method will preserve supersymmetry as well. In this paper, we will investigate the applicability of LR method in supersymmetric theories.

The paper is organized as follows: in section II, we briefly introduce the symmetry-preserving loop regularization with string-mode regulators. In sections III and IV] we will verify the supersymmetric Ward identities for the massless Wess-Zumino model and massive Wess-Zumino model [17] separately and show that the LR method indeed respects the Ward identities. As Ward identity is the reflection of symmetry in quantum level, we then arrive at the conclusion that the LR method is also a supersymmetry-preserving regularization for supersymmetric models. In section V] we consider the super Yang-Mills theory as the testing ground to explicitly demonstrate the supersymmetric Ward identity and show that the LR method does preserve supersymmetry, and meanwhile the gauge symmetry is maintained only requiring the consistency condition for logarithmic divergences. Note that the conventional dimensional regularization was shown to break the 
Ward identity in such a model[], thus an alternative check by using LR method in our present paper is nontrivial. In particular, we will demonstrate that as long as the Dirac algebra for $\gamma$ matrices are carried out in four dimensional space-time, and the shift of integration variable can be safely made, the supersymmetric Ward identities are preserved, which is actually independent of any concrete prescription of regularization methods. Namely, as long as the regularization scheme is realized in four dimensional space-time with translational invariance for momentum integrals, like the LR method, it then preserves supersymmetry. In section VI, as an explicit demonstration, we will carry out the one-loop renormalization for the massive Wess-Zumino model by using the LR method, and all the obtained results agree with the well-known non-renormalization theorem. Our conclusions and remarks are given in the last section. The detailed derivation of Majorana Feynman rules is presented in the appendix.

\section{SYMMETRY-PRESERVING LOOP REGULARIZATION}

It has been shown in [11, 12] that all one loop Feynman integrals can be evaluated into the following 1-fold ILIs by using the Feynman parameterization method:

$$
\begin{aligned}
I_{-2 \alpha} & =\int \frac{d^{4} k}{(2 \pi)^{4}} \frac{1}{\left(k^{2}-M^{2}\right)^{2+\alpha}}, \\
I_{-2 \alpha \mu \nu} & =\int \frac{d^{4} k}{(2 \pi)^{4}} \frac{k_{\mu} k_{\nu}}{\left(k^{2}-M^{2}\right)^{3+\alpha}}, \quad \alpha=-1,0,1,2, \ldots \\
I_{-2 \alpha \mu \nu \rho \sigma} & =\int \frac{d^{4} k}{(2 \pi)^{4}} \frac{k_{\mu} k_{\nu} k_{\rho} k_{\sigma}}{\left(k^{2}-M^{2}\right)^{4+\alpha}}
\end{aligned}
$$

with $I_{2}$ and $I_{0}$ corresponding to the quadratic and logarithmic divergent integrals. Where the effective mass factor $M^{2}$ is a function of the external momenta $p_{i}$, the masses of particles $m_{i}$ and the Feynman parameters.

In general, the loop momentum independent $M^{2}$ can be extended to include linear term in $k$, which can be understood as a part of the definition of the ILIs in the LR. The reason is as follows: Let $M^{2}(k)$ has the following general form including linear term in $k$

$$
M^{2}(k)=M^{2}+2 x k \cdot p
$$

with $x$ an arbitrary parameter. Then

$$
k^{2}+M^{2}(k)=k^{2}+2 x p . k+M^{2}=(k+x p)^{2}+M^{2}-p^{2}=(k+x p)^{2}+M^{2}(p)=k^{\prime 2}+M^{2}(p)
$$

with $M^{2}(p)=M^{2}-p^{2}$ which becomes independent of $k$, and $k^{\prime}=k+x p$ via translational invariance. Again the only thing must be paid attention is that one must follow the definition of ILIs to cancel out the $k^{2}$ in the numerator before regularization.

When the regularized 1-fold ILIs satisfy the following consistency conditions [11, 12]:

$$
\begin{array}{ll}
I_{2 \mu \nu}^{R}=\frac{1}{2} g_{\mu \nu} I_{2}^{R}, & I_{2 \mu \nu \rho \sigma}^{R}=\frac{1}{8}\left(g_{\mu \nu} g_{\rho \sigma}+g_{\mu \rho} g_{\nu \sigma}+g_{\mu \sigma} g_{\rho \nu}\right) I_{2}^{R}, \\
I_{0 \mu \nu}^{R}=\frac{1}{4} g_{\mu \nu} I_{0}^{R}, & I_{0 \mu \nu \rho \sigma}^{R}=\frac{1}{24}\left(g_{\mu \nu} g_{\rho \sigma}+g_{\mu \rho} g_{\nu \sigma}+g_{\mu \sigma} g_{\rho \nu}\right) I_{0}^{R} .
\end{array}
$$

the resulting loop corrections are gauge invariant. Here the superscript "R" denotes the regularized ILIs.

Note that the introduction on the concept of irreducible loop integrals (ILIs) is crucial in the loop regularization [11, 12], where it has been shown that all Feynman loop integrals can be evaluated 
to be expressed by the ILIs. From the definition of ILIs, one of the important properties is that there should be no $k^{2}$ in the numerator of loop integration, all the ILIs can be classified into the scalar type ILIs with the following loop integration

$$
\frac{1}{\left(k^{2}-M^{2}\right)^{\alpha}}
$$

and the tensor type ILIs with the following loop integration

$$
\frac{k_{\mu} k_{\nu} \cdots k_{\rho}}{\left(k^{2}-M^{2}\right)^{\alpha}}
$$

In evaluating the Feynman loop integrals into ILIs, one should always perform the Dirac algebra and Lorentz index-contraction firstly to obtain the ILIs defined by the above "simplest" forms for the one loop case (for two loop and higher loop case, see ref.[11]). Therefore, for the integration

$$
g^{\mu \nu} \cdot k_{\mu} k_{\nu} /\left(k^{2}-M^{2}\right)^{2}
$$

which should not be written as

$$
g^{\mu \nu} \cdot I_{2 \mu \nu}
$$

but it must be expressed as

$$
k^{2} /\left(k^{2}-M^{2}\right)^{2}
$$

then rewriting the $k^{2}$ in the numerator into $\left(k^{2}-M^{2}\right)+M^{2}$ so as to cancel out the first term by the denominator. Thus the above Feynman loop integration is regarded to be evaluated into the ILIs and is given by the following form before regularization

$$
g^{\mu \nu} \cdot k_{\mu} k_{\nu} /\left(k^{2}-M^{2}\right)^{2}=I_{2}+M^{2} * I_{0}
$$

From the above illustration, it is seen that in the spirit of "irreducible loop integrals" (ILIs), the integration

$$
g^{\mu \nu} \cdot k_{\mu} k_{\nu} /\left(k^{2}-M^{2}\right)^{2}
$$

is not an ILI, one should not regularize such a loop integration in the loop regularization method.

A simple regularization prescription for the ILIs was realized to yield the above consistency conditions, its procedure is that: Rotating to the four dimensional Euclidean space of momentum, replacing the loop integrating variable $k^{2}$ and the loop integrating measure $\int d^{4} k$ in the ILIs by the corresponding regularized ones $\left[k^{2}\right]_{l}$ and $\int\left[d^{4} k\right]_{l}$ :

$$
\begin{aligned}
k^{2} & \rightarrow\left[k^{2}\right]_{l} \equiv k^{2}+M_{l}^{2} \\
\int d^{4} k & \rightarrow \int\left[d^{4} k\right]_{l} \equiv \lim _{N, M_{l}^{2}} \sum_{l=0}^{N} c_{l}^{N} \int d^{4} k
\end{aligned}
$$

where $M_{l}^{2}(l=0,1, \cdots)$ may be regarded as the regulator masses for the ILIs. The regularized ILIs in the Euclidean space-time are then given by:

$$
\begin{aligned}
I_{-2 \alpha}^{R} & =i(-1)^{\alpha} \lim _{N, M_{l}^{2}} \sum_{l=0}^{N} c_{l}^{N} \int \frac{d^{4} k}{(2 \pi)^{4}} \frac{1}{\left(k^{2}+M^{2}+M_{l}^{2}\right)^{2+\alpha}} \\
I_{-2 \alpha \mu \nu}^{R} & =-i(-1)^{\alpha} \lim _{N, M_{l}^{2}} \sum_{l=0}^{N} c_{l}^{N} \int \frac{d^{4} k}{(2 \pi)^{4}} \frac{k_{\mu} k_{\nu}}{\left(k^{2}+M^{2}+M_{l}^{2}\right)^{3+\alpha}}, \quad \alpha=-1,0,1,2, \ldots \\
I_{-2 \alpha \mu \nu \rho \sigma}^{R} & =i(-1)^{\alpha} \lim _{N, M_{l}^{2}} \sum_{l=0}^{N} c_{l}^{N} \int \frac{d^{4} k}{(2 \pi)^{4}} \frac{k_{\mu} k_{\nu} k_{\rho} k_{\sigma}}{\left(k^{2}+M^{2}+M_{l}^{2}\right)^{4+\alpha}}
\end{aligned}
$$


where the coefficients $c_{l}^{N}$ are chosen to satisfy the following conditions:

$$
\lim _{N, M_{l}^{2}} \sum_{l=0}^{N} c_{l}^{N}\left(M_{l}^{2}\right)^{n}=0 \quad(n=0,1, \cdots)
$$

with the notation $\lim _{N, M_{l}^{2}}$ denoting the limit $\lim _{N, M_{R}^{2} \rightarrow \infty}$. One may take the initial conditions $M_{0}^{2}=\mu_{s}^{2}=0$ and $c_{0}^{N}=1$ to recover the original integrals in the limit $M_{l}^{2} \rightarrow \infty(l=1,2, \cdots$ ). Such a new regularization is called as Loop Regularization (LR) [11, 12]. The prescription in LR method is very similar to Pauli-Villars prescription, but two concepts are totally different as the prescription in the loop regularization is acting on the ILIs rather than on the propagators in Pauli-Villars scheme. This is why the Pauli-Villars regularization violates non-Abelian gauge symmetry, while LR method can preserve non-Abelian gauge symmetry.

As the simplest solution of eq. (2.5), taking the string-mode regulators

$$
M_{l}^{2}=\mu_{s}^{2}+l M_{R}^{2}
$$

with $l=1,2, \cdots$, the coefficients $c_{l}^{N}$ are completely determined

$$
c_{l}^{N}=(-1)^{l} \frac{N !}{(N-l) ! l !}
$$

Here $M_{R}$ may be regarded as a basic mass scale of loop regulator. It has been shown in [12] that the above regularization prescription can be understood in terms of Schwinger proper time formulation with an appropriate regulating distribution function.

With the string-mode regulators for $M_{l}^{2}$ and $c_{l}^{N}$ in above equations, the regularized ILIs $I_{2}^{R}$ and $I_{0}^{R}$ can be evaluated to the following explicit forms [11, 12]:

$$
\begin{aligned}
I_{2}^{R} & =\frac{-i}{16 \pi^{2}}\left\{M_{c}^{2}-\mu^{2}\left[\ln \frac{M_{c}^{2}}{\mu^{2}}-\gamma_{w}+1+y_{2}\left(\frac{\mu^{2}}{M_{c}^{2}}\right)\right]\right\} \\
I_{0}^{R} & =\frac{i}{16 \pi^{2}}\left[\ln \frac{M_{c}^{2}}{\mu^{2}}-\gamma_{w}+y_{0}\left(\frac{\mu^{2}}{M_{c}^{2}}\right)\right]
\end{aligned}
$$

with $\mu^{2}=\mu_{s}^{2}+M^{2}$, and

$$
\begin{aligned}
& \gamma_{w} \equiv \lim _{N}\left\{\sum_{l=1}^{N} c_{l}^{N} \ln l+\ln \left[\sum_{l=1}^{N} c_{l}^{N} l \ln l\right]\right\}=\gamma_{E}=0.5772 \cdots, \\
& y_{0}(x)=\int_{0}^{x} d \sigma \frac{1-e^{-\sigma}}{\sigma}, \quad y_{1}(x)=\frac{e^{-x}-1+x}{x} \\
& y_{2}(x)=y_{0}(x)-y_{1}(x), \quad \lim _{x \rightarrow 0} y_{i}(x) \rightarrow 0, i=0,1,2 \\
& M_{c}^{2} \equiv \lim _{N, M_{R}} M_{R}^{2} \sum_{l=1}^{N} c_{l}^{N}(l \ln l)=\lim _{N, M_{R}} M_{R}^{2} / \ln N
\end{aligned}
$$

which indicates that the $\mu_{s}$ sets an IR 'cutoff' at $M^{2}=0$ and $M_{c}$ provides an UV 'cutoff'. For renormalizable quantum field theories, $M_{c}$ can be taken to be infinity $\left(M_{c} \rightarrow \infty\right)$. In a theory without infrared divergence, $\mu_{s}$ can safely run to $\mu_{s}=0$. Actually, in the case that $M_{c} \rightarrow \infty$ and $\mu_{s}=0$, one recovers the initial integral. Also once $M_{R}$ and $N$ are taken to be infinity, the regularized theory becomes independent of the regularization prescription. Note that to evaluate the ILIs, the algebraic computing for multi $\gamma$ matrices involving loop momentum $\not k$ such as $\not k \gamma_{\mu} \not k$ should be carried out to be expressed in terms of the independent components: $\gamma_{\mu}, \sigma_{\mu \nu}, \gamma_{5} \gamma_{\mu}, \gamma_{5}$. 
We shall directly show that loop regularization is manifestly translational invariant in spite of the existence of two energy scales, which is a very important feature in applying to supersymmetric theories in this paper. To see that, we shall verify that the regularized ILIs should arrive at the same results whether the loop regularization prescription is applied before or after shifting the integration variables for momentum. For an explicit illustration, let us examine a simple logarithmic divergent Feynman integral:

$$
L=\int \frac{d^{4} k}{(2 \pi)^{4}} \frac{1}{k^{2}-m_{1}^{2}} \frac{1}{(k-p)^{2}-m_{2}^{2}}
$$

As the first step of loop regularization, we shall apply the general Feynman parameter formula

$$
\begin{aligned}
\frac{1}{a_{1}^{\alpha_{1}} a_{2}^{\alpha_{2}} \cdots a_{n}^{\alpha_{n}}}= & \frac{\Gamma\left(\alpha_{1}+\cdots+\alpha_{n}\right)}{\Gamma\left(\alpha_{1}\right) \cdots \Gamma\left(\alpha_{n}\right)} \int_{0}^{1} d x_{1} \int_{0}^{x_{1}} d x_{2} \cdots \int_{0}^{x_{n-2}} d x_{n-1} \\
& \frac{\left(1-x_{1}\right)^{\alpha_{1}-1}\left(x_{1}-x_{2}\right)^{\alpha_{2}-1} \cdots x_{n-1}^{\alpha_{n}-1}}{\left[a_{1}\left(1-x_{1}\right)+a_{2}\left(x_{1}-x_{2}\right)+\cdots+a_{n} x_{n-1}\right]^{\alpha_{1}+\cdots+\alpha_{n}}}
\end{aligned}
$$

to the Feyman integral and obtain the following integral

$$
\begin{aligned}
L & =\int \frac{d^{4} k}{(2 \pi)^{4}} \int_{0}^{1} d x \frac{1}{\left\{(1-x)\left(k^{2}-m_{1}^{2}\right)+x\left[(k-p)^{2}-m_{2}^{2}\right]\right\}^{2}} \\
& =\int \frac{d^{4} k}{(2 \pi)^{4}} \int_{0}^{1} d x \frac{1}{\left\{(k-x p)^{2}-\left[(1-x) m_{1}^{2}+x m_{2}^{2}-x(1-x) p^{2}\right]\right\}^{2}} \\
& =\int_{0}^{1} d x \int \frac{d^{4} k}{(2 \pi)^{4}} \frac{1}{\left[(k-x p)^{2}-M^{2}\right]^{2}}
\end{aligned}
$$

with $M^{2}=(1-x) m_{1}^{2}+x m_{2}^{2}-x(1-x) p^{2}$.

By making Wick rotation and applying the loop regularization prescription before shifting the integration variable, i.e., rewriting the momentum factor $(k-x p)^{2}$ into $(k-x p)^{2}=k^{2}-2 x p . k+x^{2} p^{2}$, then replacing $k^{2}$ by $k^{2}+M_{l}^{2}$, namely

$$
(k-x p)^{2}=k^{2}-2 x p . k+x^{2} p^{2} \rightarrow k^{2}+M_{l}^{2}-2 x p \cdot k+x^{2} p^{2}=(k-x p)^{2}+M_{l}^{2}
$$

we then obtain the regularized Feynman integral

$$
L^{R}=i \lim _{N, M_{l}^{2}} \sum_{l=0}^{N} c_{l}^{N} \int_{0}^{1} d x \int \frac{d^{4} k}{(2 \pi)^{4}} \frac{1}{\left[(k-x p)^{2}+M^{2}+M_{l}^{2}\right]^{2}}
$$

which becomes a well defined integral, so that we can safely shift the integration variable:

$$
L^{R}=\int_{0}^{1} d x \lim _{N, M_{l}^{2}} \sum_{l=0}^{N} c_{l}^{N} \int \frac{d^{4} k}{(2 \pi)^{4}} \frac{1}{\left(k^{2}+M^{2}+M_{l}^{2}\right)^{2}}
$$

The same result can be arrived by using the standard procedure of loop regularization with first shifting the integration variable for momentum, which yields the standard scalar type ILI

$$
L_{0}=\int_{0}^{1} d x \int \frac{d^{4} k}{(2 \pi)^{4}} \frac{1}{\left(k^{2}-M^{2}\right)^{2}}=\int_{0}^{1} d x I_{0}
$$

after applying the loop regularization prescription, the same form is reached

$$
L_{0}^{R}=i \int_{0}^{1} d x \lim _{N, M_{l}^{2}} \sum_{l=0}^{N} c_{l}^{N} \int \frac{d^{4} k}{(2 \pi)^{4}} \frac{1}{\left(k^{2}+M^{2}+M_{l}^{2}\right)^{2}} \equiv L^{R}
$$


which shown that in loop regularization method, one can safely shift the integration variables and express all the Feynman integrals in terms of ILIs before applying for the regularization prescription.

From the above explicit demonstration, it is seen that the loop regularization is indeed translational invariant. In fact, this property also allows us to eliminate the ambiguities and make a consistent calculation for the chiral anomaly even in the existence of linear divergent integral [13, 14]. The similar verification of translational invariance can be extended to the linearly and quadratically divergent integrals, which is presented in the Appendix A.

The above proof can in generally be extended to higher loops based on several theorems proved in ref.[11], especially based on the theorem I, theorem V and theorem VI over there. The theorem I is the so-called factorization theorem for overlapping divergences which states that overlapping divergences which contain divergences of sub-integrals and overall divergences in the general Feynman loop integrals become completely factorizable in the corresponding ILIs. The theorem $\mathrm{V}$ is the so-called reduction theorem for overlapping tensor type integrals which states that the general overlapping tensor type Feynman integrals of arbitrary loop graphs are eventually characterized by the overall one-fold tensor type ILIs of the corresponding loop graphs. This theorem is the key theorem for the generalization of treatments and also for the prescriptions from one loop graphs to arbitrary loop graphs. The theorem VI which is the so-called relation theorem for tensor and scalar type ILIs which states that for any fold tensor and scalar type ILIs, as long as their power counting dimension of the integrating loop momentum are the same, then the relations between the tensor and scalar type ILIs are also the same and independent of the fold number of ILIs. This theorem is crucial to extend the consistency conditions of gauge invariance from divergent one loop ILIs to higher loop ILIs.

\section{WARD IDENTITY IN MASSLESS WESS-ZUMINO MODEL}

We begin with the massless Wess-Zumino theory which is the simplest supersymmetric model. The Lagrangian is:

$$
\begin{aligned}
L= & -\frac{1}{2}\left(\partial_{\mu} A\right)^{2}-\frac{1}{2}\left(\partial_{\mu} B\right)^{2}-\frac{1}{2} \bar{\chi} \partial \chi+\frac{1}{2} F^{2}+\frac{1}{2} G^{2} \\
& +g\left[-F\left(A^{2}-B^{2}\right)+2 G A B+\bar{\chi}\left(A+i \gamma_{5} B\right) \chi\right]
\end{aligned}
$$

the action is invariant, up to a total derivative, under the global supersymmetric transformation shown below:

$$
\begin{aligned}
\delta A & =\bar{\epsilon} \chi, \delta B=-i \bar{\epsilon} \gamma_{5} \chi \\
\delta \chi & =-\bar{\epsilon} \not \partial\left(A+i \gamma_{5} B\right)+\bar{\epsilon}\left(F+i \gamma_{5} G\right), \\
\delta F & =\bar{\epsilon} \not \chi \chi, \delta G=-i \bar{\epsilon} \gamma_{5} \partial \chi \chi .
\end{aligned}
$$

Using functional technique, one can deduce that the one-particle irreducible(1PI) Green functions generating functional $\Gamma$ is invariant under the supersymmetric transformation [18]. The supersymmetric Ward identity we choose to check is involving two-point irreducible functions:

$$
\frac{\delta^{2} \Gamma}{\delta A(x) \delta A(y)} \delta_{\gamma \alpha}-\left(\partial_{y}\right)_{\gamma \beta} \frac{\delta^{2} \Gamma}{\delta \chi_{\alpha}(x) \delta \bar{\chi}_{\beta}(y)}=0
$$

This could be obtained from differentiating the equantion $\delta \Gamma=0$ by $A(x)$ and $\bar{\chi}(x)[19]$. In the momentum space, we can write it as:

$$
\Gamma_{A A}(p) \delta_{\gamma \alpha}-i(\not p)_{\gamma \beta} \Gamma_{\chi_{\alpha} \bar{\chi}_{\beta}}(p)=0
$$




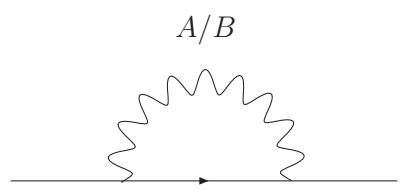

(a)

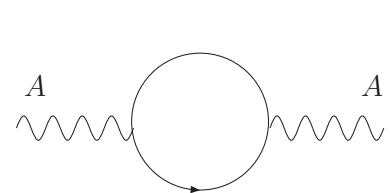

(b)

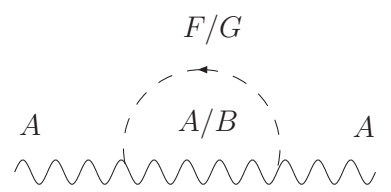

(c)

FIG. 1: Three diagrams contribute at one-loop level

at one-loop level, Feynman diagrams contribute to this identity are shown in FIG!1.

$$
\begin{aligned}
\Gamma_{\chi_{\alpha} \bar{\chi}_{\beta}}^{(a)}(p) & =2 \times 4 g^{2} \int \frac{d^{4} k}{(2 \pi)^{4}} \frac{-\gamma_{\mu} k^{\mu}}{k^{2}} \frac{-i}{(k-p)^{2}} \\
& =-i 8 g^{2} \int_{0}^{1} d x \int \frac{d^{4} l}{(2 \pi)^{4}} \frac{x \gamma_{\mu} p^{\mu}}{\left[l^{2}-x(x-1) p^{2}\right]^{2}} \\
& =-i 8 g^{2} \int_{0}^{1} d x x \gamma_{\mu} p^{\mu} I_{0}\left(x(x-1) p^{2}\right)
\end{aligned}
$$

the factor 2 appears because the wave line could be $A$ or $B$. And the factor 4 results from the fact that the fermion is a Majorana particle. We could discern this result more clearly from the Majorana Feynman rules given in the appendix. According to the Feynman rules we should calculate $\langle\chi \chi\rangle$ firstly, and then obtain the $\langle\chi \bar{\chi}\rangle$ from the relation below:

$$
\left\langle\chi_{i} \bar{\chi}_{j}\right\rangle=\left\langle\chi_{i}\left(C^{-1} \chi\right)_{j}^{T}\right\rangle=\left\langle\chi_{i} \chi_{k}\right\rangle\left(-C_{k j}^{-1}\right)
$$

The calculation of $\Gamma_{A A}$ is straight forward:

$$
\begin{aligned}
\Gamma_{A A}^{(b)}(p) & =-2 g^{2} \int \frac{d^{4} k}{(2 \pi)^{4}} \operatorname{Tr}\left[\frac{\gamma_{\mu} k^{\mu}}{k^{2}} \frac{\gamma_{v}\left(k^{\nu}-p^{\nu}\right)}{(k-p)^{2}}\right] \\
& =8 g^{2} \int \frac{d^{4} l}{(2 \pi)^{4}}\left(\int_{0}^{1} d x \frac{x p^{2}}{\left[l^{2}-x(x-1) p^{2}\right]^{2}}-\frac{1}{l^{2}}\right) \\
& =8 g^{2}\left(\int_{0}^{1} d x x p^{2} I_{0}\left(x(x-1) p^{2}\right)-I_{2}(0)\right) \\
\Gamma_{A A}^{(c)}(p) & =2 \times 4 g^{2} \int \frac{d^{4} l}{(2 \pi)^{4}} \frac{1}{l^{2}}=8 g^{2} I_{2}(0)
\end{aligned}
$$

We can see immediately that the Ward identity (3.5) is satisfied because the integrands cancel out. To arrive at above results we have only carried out Dirac algebra for $\gamma$ matrices in the four dimensional space-time and make the shift of the integration variables. As these operations are all rational in a four dimensional well-defined loop regularization method, thus we conclude that at one-loop level the LR method indeed preserves supersymmetric Ward identity in this simple model.

\section{WARD IDENTITY IN MASSIVE WESS-ZUMINO MODEL}

We are examining another supersymmetric model. The procedure is similar to what we have done in the above massless model. The Lagrangian of massive Wess-Zumino model is:

$$
\begin{aligned}
L= & -\frac{1}{2}\left(\partial_{\mu} A\right)^{2}-\frac{1}{2}\left(\partial_{\mu} B\right)^{2}-\frac{1}{2} \bar{\chi} \not \partial \chi+\frac{1}{2} F^{2}+\frac{1}{2} G^{2}+m\left(A F-B G-\frac{1}{2} \bar{\chi} \chi\right) \\
& +g\left[-F\left(A^{2}-B^{2}\right)+2 G A B+\bar{\chi}\left(A+i \gamma_{5} B\right) \chi\right]
\end{aligned}
$$




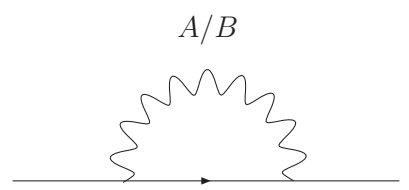

(a)

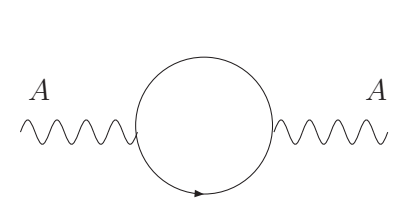

(b)

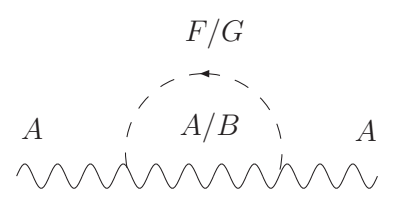

(c)

FIG. 2: The same as massless case

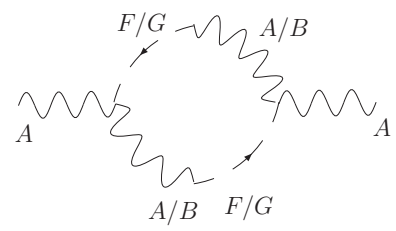

$(d)$

FIG. 3: Additional diagram contributing to $\Gamma_{A A}$.
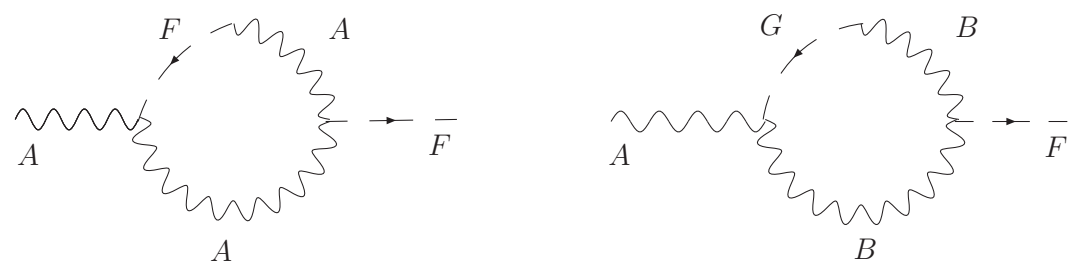

FIG. 4: Two diagrams contributing to $\Gamma_{A F}$

It is different from the massless case with the mass term $m\left(A F-B G-\frac{1}{2} \bar{\chi} \chi\right)$. In this model bosons and fermions have equal masses as demanded by supersymmetry. In section VI, we will explicitly show that the radiative corrections do not violate such an equality. The supersymmetric transformation of component fields are the same as Eq.(3.3). Following the same procedure, the two-point Ward identity of this model is extended to be[20]:

$$
\Gamma_{A A}(p) \delta_{\gamma \alpha}-i(\not p)_{\gamma \beta} \Gamma_{\chi_{\alpha} \bar{\chi}_{\beta}}(p)+i(\not p)_{\gamma \alpha} \Gamma_{A F}(p)=0
$$

At one-loop level, the diagrams which contribute to this supersymmetric Ward identity are shown in FIG 2, FIG 3 and FIG,4.

It is easy to show that two diagrams in Fig.(4) contribute to $\Gamma_{A F}$ and their contributions cancel each other:

$$
\Gamma_{A F}=4 g^{2} \int \frac{d^{4} k}{(2 \pi)^{4}}\left[\frac{1}{k^{2}-m^{2}} \frac{m}{(k-p)^{2}-m^{2}}+\frac{1}{k^{2}-m^{2}} \frac{-m}{(k-p)^{2}-m^{2}}\right]=0
$$

The calculations of other diagrams are straightforward, we just present the final results as follows:

$$
\begin{aligned}
\Gamma_{\chi_{\alpha} \bar{\chi}_{\beta}}^{(a)}(p) & =4 g^{2} \int \frac{d^{4} k}{(2 \pi)^{4}}\left[\frac{-i \gamma_{\mu} k^{\mu}+m}{k^{2}-m^{2}} \frac{1}{(k-p)^{2}-m^{2}}+\left(i \gamma_{5}\right) \frac{-i \gamma_{\mu} k^{\mu}+m}{k^{2}-m^{2}}\left(i \gamma_{5}\right) \frac{1}{(k-p)^{2}-m^{2}}\right] \\
& =8 g^{2} \int \frac{d^{4} l}{(2 \pi)^{4}} \int_{0}^{1} d x \frac{-i x \gamma_{\mu} p^{\mu}}{\left[l^{2}-x(x-1) p^{2}-m^{2}\right]^{2}}
\end{aligned}
$$




$$
\begin{aligned}
& =8 g^{2} \int_{0}^{1} d x\left(-i x \gamma_{\mu} p^{\mu}\right) I_{0}\left(x(x-1) p^{2}+m^{2}\right) \\
\Gamma_{A A}^{(b)}(p) & =-2 g^{2} \int \frac{d^{4} k}{(2 \pi)^{4}} \operatorname{Tr}\left[\frac{-i \gamma_{\mu} k^{\mu}+m-i \gamma_{v}\left(k^{\nu}-p^{\nu}\right)+m}{k^{2}-m^{2}} \frac{1}{(k-p)^{2}-m^{2}}\right] \\
& =8 g^{2} \int \frac{d^{4} k}{(2 \pi)^{4}} \int_{0}^{1} d x\left[\frac{2\left[m^{2}+x(1-x) p^{2}\right]}{\left[l^{2}-x(x-1) p^{2}-m^{2}\right]}-\frac{1}{\left[l^{2}-x(x-1) p^{2}-m^{2}\right]^{2}}\right] \\
& =8 g^{2} \int_{o}^{1} d x\left[I_{2}\left(x(x-1) p^{2}+m^{2}\right)-2\left[m^{2}+x(1-x) p^{2}\right] I_{0}\left(x(x-1) p^{2}+m^{2}\right)\right] \\
\Gamma_{A A}^{(c)}(p) & =2 \times 4 g^{2} \int \frac{d^{4} k}{(2 \pi)^{4}} \frac{-k^{2}}{k^{2}-m^{2}} \frac{1}{(k-p)^{2}-m^{2}} \\
& =8 g^{2} \int \frac{d^{4} l}{(2 \pi)^{4}} \int_{0}^{1} d x\left[\frac{-1}{\left[l^{2}-x(x-1) p^{2}-m^{2}\right]}+\frac{m^{2}+x(1-2 x) p^{2}}{\left[l^{2}-x(x-1) p^{2}-m^{2}\right]^{2}}\right] \\
& =8 g^{2} \int_{o}^{1} d x\left[-I_{2}\left(x(x-1) p^{2}+m^{2}\right)+\left[m^{2}+x(1-2 x) p^{2}\right] I_{0}\left(x(x-1) p^{2}+m^{2}\right)\right] \\
\Gamma_{A A}^{(d)}(p) & =2 \times 4 g^{2} \int \frac{d^{4} k}{(2 \pi)^{4}} \frac{m}{k^{2}-m^{2}} \frac{m}{(k-p)^{2}-m^{2}} \\
& =8 g^{2} \int_{\frac{m^{2}}{(2 \pi)^{4}} \int_{0}^{1} d x \frac{d^{4}}{\left[l^{2}-x(x-1) p^{2}-m^{2}\right]^{2}}} \\
& =8 g^{2} \int_{0}^{1} m^{2} I_{0}\left(x(x-1) p^{2}+m^{2}\right)
\end{aligned}
$$

Adding all the contributions together, we can see that the integrands cancel out and the supersymmetric Ward identity holds. Again, to arrive at above results we have only performed Dirac algebra for $\gamma$ matrices in the four dimensional space-time and make the shift of the integration variables. It further shows that in the massive Wess-Zumino model the LR method can preserve supersymmetry as well.

\section{WARD IDENTITY IN SUPERSYMMETRIC GAUGE THEORY}

Let us consider a more complicated case, i.e., the supersymmetric Yang-Mills theory. This model involves supersymmetry as well as gauge symmetry. In the Wess-Zumino gauge, the Lagrangian (with source terms) can be writen as:

$$
L=-\frac{1}{4}\left(F_{\mu \nu}^{a}\right)^{2}-\frac{1}{2}\left(\partial^{\mu} A_{\mu}^{a}\right)+C^{* a} \partial D^{a b} C^{b}-\frac{1}{2} \bar{\lambda}^{a} D^{a b} \lambda^{b}+\frac{1}{2} D_{a}^{2}+J^{a \mu} A_{\mu}^{a}+\bar{J}^{a} \lambda^{a}+j_{D}^{a} D^{a}
$$

where $\lambda^{a}$ is a Majorana spinor and $D^{a}$ is the auxiliary field. Similarly, the supersymmetric Ward identity is derived by considering the functional variation of the Green function generating functional under an infinitesimal supersymmetric transformation. All the fields transform as follows:

$$
\begin{aligned}
\delta A_{\mu}^{a} & =-\bar{\epsilon} \gamma_{\mu} \lambda^{a}, \\
\delta \lambda^{a} & =\sigma^{\mu \nu} F_{\mu \nu}^{a} \epsilon+i \gamma_{5} D^{a} \epsilon, \\
\delta D^{a} & =\bar{\epsilon} i \gamma_{5} D^{a b} \lambda^{b}
\end{aligned}
$$

which lead to the following supersymmetric Ward identity [6, 21]:

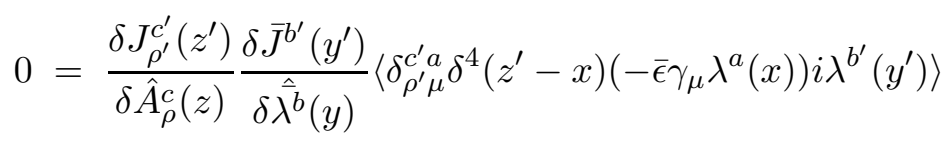




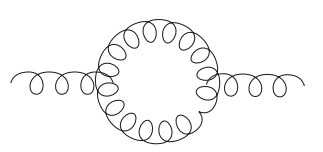

(1)

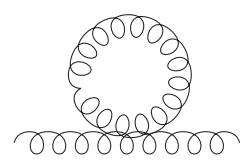

(2)

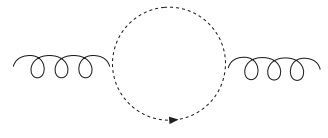

(3)

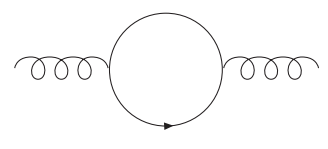

(4)

FIG. 5: the 1st term

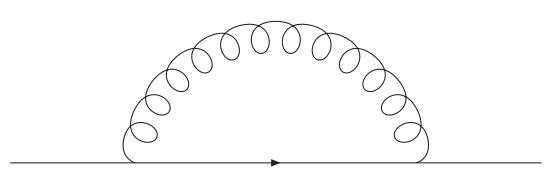

(5)

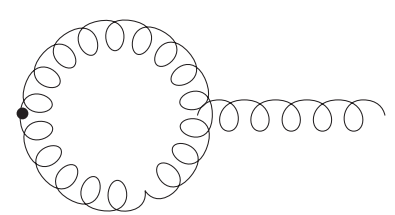

(6)

FIG. 6: the 2nd term

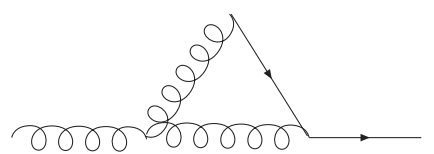

(7)

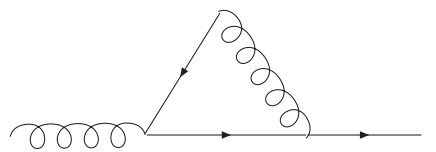

(8)

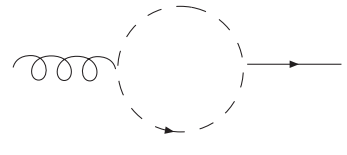

(9)

FIG. 7: the 3rd and 4th terms

$$
\begin{aligned}
& +\frac{\delta J_{\rho^{\prime}}^{c^{\prime}}\left(z^{\prime}\right)}{\delta \hat{A}_{\rho}^{c}(z)} \frac{\delta{\overline{J^{b}}}^{b^{\prime}}\left(y^{\prime}\right)}{\delta \hat{\bar{\lambda}}^{b}(y)}\left\langle\delta^{b^{\prime} a} \delta^{4}\left(y^{\prime}-x\right) i A_{\rho^{\prime}}^{c^{\prime}}\left(z^{\prime}\right) \sigma^{\mu \nu} F_{\mu \nu}^{a}(x) \epsilon\right\rangle \\
& +\frac{\delta J_{\rho^{\prime}}^{c^{\prime}}\left(z^{\prime}\right)}{\delta \hat{A}_{\rho}^{c}(z)} \frac{\delta{\overline{J^{\prime}}}^{b^{\prime}}\left(y^{\prime}\right)}{\delta \hat{\bar{\lambda}}^{b}(y)}\left\langle\partial \cdot A^{a}(\mathrm{x}) \bar{\epsilon} \partial \lambda^{a}(x) i \lambda^{b^{\prime}}\left(y^{\prime}\right) i A_{\rho^{\prime}}^{c^{\prime}}\left(z^{\prime}\right)\right\rangle \\
& +\frac{\delta{J^{\prime}}^{c^{\prime}}\left(z^{\prime}\right)}{\delta \hat{A}_{\rho}^{c}(z)} \frac{\delta{\overline{J^{\prime}}}^{b^{\prime}}\left(y^{\prime}\right)}{\delta \hat{\bar{\lambda}}^{b}(y)}\left\langle i \lambda^{b^{\prime}}\left(y^{\prime}\right) i A_{\rho^{\prime}}^{c^{\prime}}\left(z^{\prime}\right)\left(\partial_{\mu} C^{* a}\right) f^{a e f} \bar{\epsilon} \gamma_{\mu} \lambda^{e}(\mathrm{x}) C^{f}(x)\right\rangle \\
& +\frac{\delta^{2} \bar{J}^{b^{\prime}}\left(y^{\prime}\right)}{\delta \hat{A}_{\rho}^{c}(z) \delta \hat{\overline{\lambda^{b}}}(y)}\left\langle\partial \cdot A^{a}(\mathrm{x}) \bar{\epsilon} \not \lambda^{a}(x) i \lambda^{b^{\prime}}\left(y^{\prime}\right)\right\rangle \\
& +\frac{\delta^{2} \bar{J}^{b^{\prime}}\left(y^{\prime}\right)}{\delta \hat{A}_{\rho}^{c}(z) \delta \hat{\overline{\lambda^{b}}}(y)}\left\langle i \lambda^{b^{\prime}}\left(y^{\prime}\right)\left(\partial_{\mu} C^{* a}\right) f^{a e f} \bar{\epsilon} \gamma_{\mu} \lambda^{e}(\mathrm{x}) C^{f}(x)\right\rangle
\end{aligned}
$$

here the notation $\langle\ldots\rangle$ represents connected Green functions and the integration over $x, y^{\prime}, z^{\prime}$ are abbreviated. At the tree level, only the 1st, 2nd and 3rd terms in the above equation contribute, one can easily verify that the identity holds. At the one-loop level, only the 1st, 2nd, 3rd and 4th terms contribute, all the diagrams to this order are shown in FIG $[5$, FIG 6 and FIG.7.

We would like to point out that the first line of eq. (5.3) is exactly the self-energy function of the gauge boson at one-loop level, it can be seen from the relation below:

$$
\frac{\delta J_{\rho^{\prime}}^{c^{\prime}}\left(z^{\prime}\right)}{\delta \hat{A}_{\rho}^{c}(z)}=\Gamma_{A_{\rho^{\prime}}^{c^{\prime}} A_{\rho}^{c}}\left(z-z^{\prime}\right)
$$


Gauge symmetry requires this term to be transverse.

We now turn to the calculation of each term in the Ward identity, and choose $\xi=1$ for simplicity.

$$
\begin{aligned}
\Pi_{\mu \nu}^{(1)}= & -\frac{1}{2} g^{2} C_{a c d} C_{b d c} \int \frac{d^{4} q}{(2 \pi)^{4}} \frac{1}{q^{2}(q+p)^{2}}\left[10 q_{\mu} q_{\nu}+5\left(p_{\mu} q_{\nu}+p_{\nu} q_{\mu}\right)\right. \\
& \left.-2 p_{\mu} p_{\nu}+\left(5 p^{2}+2 p \cdot q+2 q^{2}\right) g_{\mu \nu}\right] \\
= & -\frac{1}{2} g^{2} C_{a c d} C_{b d c} \int d x \int \frac{d^{4} l}{(2 \pi)^{4}} \frac{\left[10 l_{\mu} l_{\nu}+10 x^{2} p_{\mu} p_{\nu}-7 p_{\mu} p_{\nu}+4 p^{2} g_{\mu \nu}+2\left(l^{2}+x^{2} p^{2}\right) g_{\mu \nu}\right]}{\left[l^{2}-x(x-1) p^{2}\right]^{2}} \\
= & -\frac{1}{2} g^{2} C_{a c d} C_{b d c} \\
& \int d x\left[\left(10 I_{2 \mu \nu}+2 g_{\mu \nu} I_{2}\right)+\left(10 x^{2} p_{\mu} p_{\nu}-7 p_{\mu} p_{\nu}+4 p^{2} g_{\mu \nu}+2 x(2 x-1) p^{2} g_{\mu \nu}\right) I_{0}\right] \\
\Pi_{\mu \nu}^{(2)}= & g^{2} C_{a c d} C_{b d c} \int \frac{d^{4} q}{(2 \pi)^{4}} \frac{3 g_{\mu \nu}}{q^{2}}=g^{2} C_{a c d} C_{b d c} \int d x \int \frac{d^{4} l}{(2 \pi)^{4}} \frac{3 g_{\mu \nu}\left(l^{2}+x^{2} p^{2}\right)}{\left[l^{2}-x(x-1) p^{2}\right]^{2}} \\
= & g^{2} C_{a c d} C_{b d c} \int d x\left[3 g_{\mu \nu} I_{2}+3 x(x-1) p^{2} g_{\mu \nu} I_{0}\right] \\
\Pi_{\mu \nu}^{(3)}= & g^{2} C_{a c d} C_{b d c} \int \frac{d^{4} q}{(2 \pi)^{4}} \frac{p_{\mu} q_{\nu}+q_{\mu} q_{\nu}}{q^{2}(q+p)^{2}} \\
= & g^{2} C_{a c d} C_{b d c} \int d x \int \frac{d^{4} l}{(2 \pi)^{4}} \frac{1}{\left[l^{2}-x(x-1) p^{2}\right]^{2}}\left[-\frac{1}{2} p_{\mu} p_{\nu}+l_{\mu} l_{\nu}+x^{2} p_{\mu} p_{\nu}\right] \\
= & g^{2} C_{a c d} C_{b d c} \int d x\left[I_{2 \mu \nu}+\left(x^{2}-\frac{1}{2}\right) p_{\mu} p_{\nu} I_{0}\right] \\
= & -g^{2} C_{a c d} C_{b c d} \int d x\left[\left(4 x^{2}-3\right)\left(p^{2} g_{\mu \nu}-p_{\mu} p_{\nu}\right) I_{0}-4 I_{2 \mu \nu}+2 g_{\mu \nu} I_{2}\right] \\
\Pi_{\mu \nu}^{(1+2+3)} & (5.7)
\end{aligned}
$$

Notice that in supersymmetric Yang-Mills theory, the fermions are massless and belong to the adjoint representation of gauge group as required by the fermion-boson symmetry. Then,

$$
\begin{aligned}
\Pi_{\mu \nu}^{(4)} & =-g^{2} 4 \operatorname{tr}\left[T_{a} T_{b}\right] \int \frac{d^{4} q}{(2 \pi)^{4}} \frac{(p+q)_{\mu} q_{\nu}+(p+q)_{\nu} q_{\mu}-\left(q^{2}+q \cdot p\right) g_{\mu \nu}}{q^{2}(q+p)^{2}} \\
& =g^{2} C_{a c d} C_{b c d} \int d x \int \frac{d^{4} l}{(2 \pi)^{4}}\left[\frac{\left(4 x^{2}-2\right)\left(p^{2} g_{\mu \nu}-p_{\mu} p_{\nu}\right)-4 l_{\mu} l_{\nu}}{\left[l^{2}-x(x-1) p^{2}\right]^{2}}+\frac{2 g_{\mu \nu}}{\left[l^{2}-x(x-1) p^{2}\right]}\right] \\
& =g^{2} C_{a c d} C_{b c d} \int d x\left[\left(4 x^{2}-2\right)\left(p^{2} g_{\mu \nu}-p_{\mu} p_{\nu}\right) I_{0}-4 I_{2 \mu \nu}+2 g_{\mu \nu} I_{2}\right]
\end{aligned}
$$

Adding the four terms together, we obtain the self-energy of the gauge boson which is gauge covariant:

$$
\Pi_{\mu \nu}=g^{2} C_{a c d} C_{b c d}\left(p^{2} g_{\mu \nu}-p_{\mu} p_{\nu}\right) \int d x I_{0}
$$

It is seen that the transverse condition of $\Pi_{\mu \nu}$ is satisfied in supersymmetric model with the Feynman gauge $\xi=1$ gauge. The reason is that the quadratical divergences which will potentially break the transverse condition cancel out in the supersymmetric model. In fact, the cancelation of quadratical divergences is a general feature of supersymmetric field theories, it is also one of the motivations to propose supersymmetry. In other word, if one wants to break supersymmetry but still maintain the gauge symmetry, there are several ways to realize that, for instance, give a mass to the fermion. In this case, the quadratical divergences do not cancel out automatically and they may destroy the transverse condition unless they can be regularized via an appropriate 
regularization method to satisfy the consistency conditions [11]. As shown in [11, 12] the LR method is competent in this case.

Note that here we have carried out the calculation in the Feynman gauge with $\xi=1$ for simplicity. In the general $\xi$ gauge, there is a term which could break the transverse condition if the regularization scheme does not satisfy the consistency condition for the logarithmic divergences, the term is in proportion to

$$
(\xi-1) *\left(a_{0}-1\right)
$$

with $a_{0}$ being defined via logarithmic divergent $I_{0 \mu \nu}=\frac{1}{4} a_{0} g_{\mu \nu} I_{0}$. In the Feynman gauge this term vanishes due to $\xi=1$. In the general $\xi$ gauge, it remains to require the regularization scheme satisfy the consistency condition for logarithmic divergent part, i.e., $a_{0}=1$, so that the transverse condition in gauge boson self-energy can hold.

And the fermion self-energy diagram is given by:

$$
\begin{aligned}
2 \sigma^{\alpha \beta} p_{\beta} \Gamma_{\lambda \lambda}^{(5)} & =-2 \sigma^{\alpha \beta} p_{\beta} \int \frac{d^{4} q}{(2 \pi)^{4}} C_{a c d} C_{b c d} \times g^{2} \gamma^{\mu} \frac{i}{(\not d+\not p)} \gamma^{\nu} \frac{-i}{q^{2}} g_{\mu \nu} \\
& =g^{2} C_{a c d} C_{b c d}\left(p^{2} \gamma^{\alpha}-\not p p^{\alpha}\right) \int \frac{d^{4} l}{(2 \pi)^{4}} \int d x \frac{1}{\left[l^{2}-x(x-1) p^{2}\right]^{2}} \\
& =g^{2} C_{a c d} C_{b c d}\left(p^{2} \gamma^{\alpha}-\not p p^{\alpha}\right) \int d x I_{0}
\end{aligned}
$$

There are two diagrams from the second term of Eq.(5.3). The non-linear part of $F_{\mu \nu}^{a}($ Fig $6(6))$ gives rise to the contribution:

$$
\begin{aligned}
\not p \Pi_{A}^{(6)}(p)= & \not p g^{2} C_{a c d} C_{b c d} \sigma^{\lambda \nu} \int \frac{d^{4} q}{(2 \pi)^{4}} \frac{-i g_{\mu \nu}}{q^{2}} \frac{-i g_{\rho \lambda}}{(q+p)^{2}}\left[g^{\tau \rho}(p-q)^{\mu}+g^{\rho \mu}(2 q+p)^{\tau}\right. \\
& \left.-g^{\mu \tau}(2 p+q)^{\rho}\right] \\
= & \frac{3}{2} g^{2} C_{a c d} C_{b c d}\left(\not p p^{\tau}-p^{2} \gamma^{\tau}\right) \int \frac{d^{4} l}{(2 \pi)^{4}} \int d x \frac{1}{\left[l^{2}-x(x-1) p^{2}\right]^{2}} \\
= & \frac{3}{2} g^{2} C_{a c d} C_{b c d}\left(\not p p^{\tau}-p^{2} \gamma^{\tau}\right) \int d x I_{0}
\end{aligned}
$$

To proceed, we consider the rest diagrams coming from the third and fourth terms of Eq.(5.3).

$$
\begin{aligned}
\Gamma_{\nu}^{(7)}= & \int \frac{d^{4} q}{(2 \pi)^{4}}\left[g C_{a c d} \gamma^{\rho}\right] i(\not p+\not q) \frac{i}{(\not d+\not p)}\left[-i(p+q)^{\lambda} \frac{-i g_{\mu \lambda}}{(p+q)^{2}}\right] \\
& \times\left[g^{\nu k}(q-p)^{\mu}-g^{k \mu}(2 q+p)^{\nu}+g^{\mu \nu}(2 p+q)^{k}\right]\left(-i g C_{b d c}\right) \frac{-i g_{\rho k}}{q^{2}} \\
= & g^{2} C_{a c d} C_{b c d} \int d x \int \frac{d^{4} l}{(2 \pi)^{4}} \gamma_{\mu}\left[\frac{g^{\nu \mu}\left(l^{2}+x^{2} p^{2}\right)-g^{\nu \mu} p^{2}-l^{\mu} l^{\nu}-x^{2} p^{\mu} p^{\nu}+p^{\mu} p^{\nu}}{\left[l^{2}-x(x-1) p^{2}\right]^{2}}\right] \\
= & g^{2} C_{a c d} C_{b c d} \int d x \gamma_{\mu}\left[g^{\mu \nu} I_{2}-I_{2}^{\mu \nu}+\left(x(2 x-1) p^{2}-g^{\mu \nu} p^{2}+\left(1-x^{2}\right) p^{\mu} p^{\nu}\right) I_{0}\right] \\
\Gamma_{\nu}^{(8)}= & \int \frac{d^{4} q}{(2 \pi)^{4}}\left[g C_{d a c} \gamma^{\mu}\right] \frac{i}{(\not d+\not p)}\left[g C_{b d c} \gamma^{\nu}\right] i \frac{i q}{\not q} \frac{-i g_{\mu \lambda}}{q^{2}}\left(i q^{\lambda}\right) \\
= & -g^{2} C_{a c d} C_{b c d} \int d x \int \frac{d^{4} l}{(2 \pi)^{4}} \gamma^{\nu}\left[\frac{l^{2}+x^{2} p^{2}-\frac{1}{2} p^{2}}{\left[l^{2}-x(x-1) p^{2}\right]^{2}}\right] \\
= & -g^{2} C_{a c d} C_{b c d} \int d x \gamma^{\nu}\left[I_{2}+\left(x(2 x-1) p^{2}-\frac{1}{2} p^{2}\right) I_{0}\right]
\end{aligned}
$$




$$
\begin{aligned}
\Gamma_{\nu}^{(9)} & =-g^{2} C_{a c d} C_{b c d} \int \frac{d^{4} q}{(2 \pi)^{4}} \frac{i q^{\rho} \gamma_{\rho}}{q^{2}} i(q+p)^{\nu} \frac{1}{(q+p)^{2}} \\
& =g^{2} C_{a c d} C_{b c d} \int d x \int \frac{d^{4} q}{(2 \pi)^{4}} \gamma_{\rho}\left[\frac{l^{\nu} l^{\rho}+x^{2} p^{\nu} p^{\rho}-\frac{1}{2} p^{\nu} p^{\rho}}{\left[l^{2}-x(x-1) p^{2}\right]^{2}}\right] \\
& =g^{2} C_{a c d} C_{b c d} \int d x \gamma_{\rho}\left[I_{2}^{\nu \rho}+\left(x^{2}-\frac{1}{2}\right) p^{\nu} p^{\rho} I_{0}\right]
\end{aligned}
$$

the total contributions of three diagrams are found to be:

$$
-\frac{1}{2} g^{2} C_{a c d} C_{b c d}\left(p^{2} \gamma^{\nu}-\not p p^{\nu}\right) \int d x I_{0}
$$

After taking into account of ' $i$ ' factors from the formula, and adding all the terms together, the integrands cancel out again, which demonstrates that the supersymmetric Ward identity does hold. To arrive this conclusion, we have only used the properties of four dimensional $\gamma$ matrices and translational invariance of momentum integrals. This implies that the LR method can indeed preserve supersymmetry. The gauge symmetry holds only requiring the consistency condition for logarithmic divergent part due to the cancelation of quadratical divergences in supersymmetrypreserving regularization method. In general, to preserve gauge symmetry in non-supersymmetric models, it needs the consistency conditions for both quadratic and logarithmic divergences for the regularized ILIs. So far, we can conclude that the LR method preserves not only non-Abelian gauge symmetry, but also supersymmetry.

\section{RENORMALIZATION OF MASSIVE WESS-ZUMINO MODEL}

In the previous sections we have shown that the LR method can respect supersymmetric Ward identities in several models including supersymmetric gauge theory, which implies that the LR method is viable in supersymmetric theories. While in the above applications, we have only used the main features of the LR method, namely the LR method is realized in four dimensions with translational invariance of momentum. In this section we shall apply the LR method to manifestly perform one-loop renormalization for the massive Wess-Zumino model. We choose such a model as a testing ground because it is fairly simple and well-known. The model was shown to be renormalizable to all orders in perturbation theory 18] by using higher derivative regularization. The same conclusion can easily be obtained in the superspace formalism, where supergraph Feynman rules of superfields greatly simplify the calculations. For our purpose, we will use the component fields formalism to renormalize the theory. This is because the superspace formalism maintains supersymmetry in a manifest way, which is not suitable for checking the consistency of a specific regularization scheme in preserving supersymmetry. On the other hand, for the physically interesting case of broken supersymmetry, it is usually preferred to work with component fields.

The action of massive Wess-Zumino model is:

$$
S_{W Z}=\frac{1}{4} \int d^{4} x d^{2} \Theta\left(\frac{1}{8} \Phi \bar{D} \Phi-\frac{1}{2} m \Phi^{2}-\frac{1}{3} g \Phi^{3}\right)+h . c .
$$

where $\Phi(x, \Theta, \bar{\Theta})$ is a chiral superfield. In terms of component fields the Lagrangian can be written as:

$$
\begin{aligned}
L= & \frac{1}{2}\left(\partial_{\mu} A \partial^{\mu} A+\partial_{\mu} B \partial^{\mu} B+i \bar{\chi} \not \partial \chi+F^{2}+G^{2}\right)-m\left(A F+B G+\frac{1}{2} \bar{\chi} \chi\right) \\
& -g\left[\left(A^{2}-B^{2}\right) F+2 A B G+\bar{\chi}\left(A-i \gamma_{5} B\right) \chi\right]
\end{aligned}
$$


The notions used here are slightly different from which in section IV. It is seen that the fields $F$ and $G$ have no dynamical terms, they are auxiliary fields and can be integrated out, which is equivalent to eliminate them from the Lagrangian by using the equations of motions. In fact, in the building of phenomenological supersymmetric model the auxiliary fields are eliminated by

$$
\begin{aligned}
& F=m A+g\left(A^{2}-B^{2}\right) \\
& G=m B+2 g A B
\end{aligned}
$$

Thus the Lagrangian can be written as:

$$
\begin{aligned}
L= & \frac{1}{2}\left(\partial_{\mu} A \partial^{\mu} A-m^{2} A^{2}\right)+\frac{1}{2}\left(\partial_{\mu} B \partial^{\mu} B-m^{2} B^{2}\right)+\frac{1}{2} \bar{\chi}(i \not \partial-m) \chi \\
& -m g A\left(A^{2}+B^{2}\right)-g \bar{\chi}\left(A-i \gamma_{5} B\right) \chi-\frac{1}{2} g^{2}\left(A^{2}+B^{2}\right)^{2}
\end{aligned}
$$

which is the Lagrangian to be renormalized by using the LR method. The Lagrangian contains one scalar particle $A$, one pesudoscalar particle $B$ and one Majorana fermion $\chi$ with equal masses $m$.

Before proceeding, we will first check what supersymmetry can tell us about the renormalization of massive Wess-Zumino model. The answer can easily be yielded in the superfield formalism based on the powerful supergraph technique. In the superfield formalism, the non-renormalization theorem implies that up to any order of the perturbative series only the first term (dynamical term) in $\mathrm{Eq}(6.1)$ needs a counterterm due to the supersymmetry. Namely, after renormalization the action gets the following form:

$$
\begin{aligned}
S_{W Z} & =\frac{1}{4} \int d^{4} x d^{2} \Theta\left(\frac{1}{8} \Phi \bar{D} \Phi-\frac{1}{2} m \Phi^{2}-\frac{1}{3} g \Phi^{3}+\frac{1}{8} \delta \Phi \bar{D} \Phi\right)+\text { h.c. } \\
& =\frac{1}{4} \int d^{4} x d^{2} \Theta\left(\frac{1}{8} Z \Phi \bar{D} \Phi-\frac{1}{2} \frac{m}{Z} Z \Phi^{2}-\frac{1}{3} \frac{g}{Z^{3 / 2}} Z^{3 / 2} \Phi^{3}\right)+\text { h.c. }
\end{aligned}
$$

Where the $\delta$ term with $\delta=Z-1$ is a logarithmically divergent counterterm, and $Z^{1 / 2}$ is the renormalization constant of the superfield. In terms of component fields, the equations of motion for $F$ and $G$ fields now become:

$$
\begin{aligned}
F & =\frac{1}{Z}\left[m A+g\left(A^{2}-B^{2}\right)\right] \\
G & =\frac{1}{Z}(m B+2 g A B)
\end{aligned}
$$

After eleminating the auxiliary fields, it them leads to the renormalized Lagrangian:

$$
\begin{aligned}
L= & \frac{1}{2} Z\left(\partial_{\mu} A \partial^{\mu} A-\left(\frac{m}{Z}\right)^{2} A^{2}\right)+\frac{1}{2} Z\left(\partial_{\mu} B \partial^{\mu} B-\left(\frac{m}{Z}\right)^{2} B^{2}\right)+\frac{1}{2} Z \bar{\chi}\left(i \not \partial-\frac{m}{Z}\right) \chi \\
& -\frac{m}{Z} \frac{g}{Z^{3 / 2}} Z^{3 / 2} A\left(A^{2}+B^{2}\right)-\frac{g}{Z^{3 / 2}} Z^{3 / 2} \bar{\chi}\left(A-i \gamma_{5} B\right) \chi-\frac{1}{2}\left(\frac{g}{Z^{3 / 2}}\right)^{2} Z^{2}\left(A^{2}+B^{2}\right)^{2}
\end{aligned}
$$

Which shows that the renormalizations of fields, mass and coupling constant must satisfy:

$$
\phi_{\text {bare }}=Z^{1 / 2} \phi ; \quad m_{\text {bare }}=Z^{-1} m ; \quad g_{\text {bare }}=Z^{-3 / 2} g \text {. }
$$

with $\phi=A, B, \chi$. We may summarize the features of the model: i). This model is renormalizable, and after renormalization all the vertexes are remained to be only one coupling constant. ii). The fields, mass and coupling constant share a common renormalization constant, which only contains logarithmical divergence. The cancellation of quadratical divergence is a general feature of all 
supersymmetric theories. iii). As required by supersymmetry, the masses of bosons still equal to the mass of fermion after renormalization.

Let us now make a detailed calculation for one-loop renormalization by using the LR Method. The Feynman rules of Lagrangian(Eq.(6.5) ) are listed in the appendix, there are 7 types of vertices. What we are going to demonstrate is that after renormalization all these 7 types of vertices will get the same renormalized coupling constant, and all the renormalization constants satisfy Eq.(6.10). It is easy to verify that one-loop contributions to $\langle A\rangle,\langle B\rangle,\langle A B\rangle,\langle A A B\rangle,\langle B B B\rangle,\langle A A A B\rangle$, $\langle A B B B\rangle$ are vanishing. The rest of divergent diagrams at one-loop level are shown in FIG 8 , the permutation graphs are not presented for simplicity.
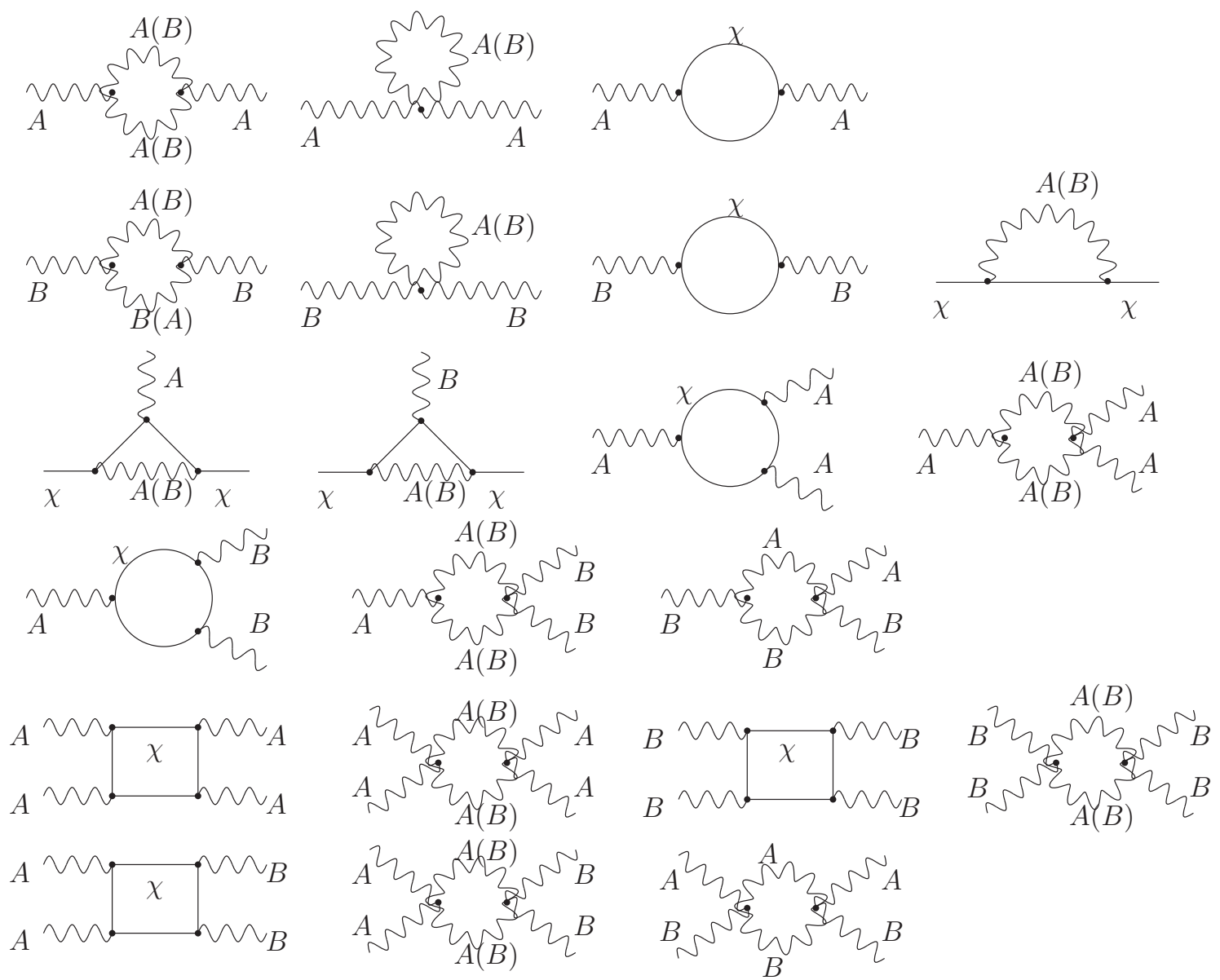

FIG. 8: non-vanishing one-loop divergent graphs in massive Wess-Zumino model

The field strength and mass renormalizations of filed $A$ can be obtained from the calculations of two point Green function $\langle A A\rangle$. Five diagrams can contribute to $\langle A A\rangle$, the total contribution is found to be:

$$
\begin{aligned}
L_{\langle A A\rangle}= & \frac{1}{2} \int \frac{d^{4} k}{(2 \pi)^{4}}\left[(-6 i m g)^{2}+(-2 i m g)^{2}\right] \frac{i}{k^{2}-m^{2}} \frac{1}{(k+p)^{2}-m^{2}} \\
& +\frac{1}{2} \int \frac{d^{4} k}{(2 \pi)^{4}}\left(-12 i g^{2}-4 i g^{2}\right) \frac{i}{k^{2}-m^{2}} \\
& -\frac{1}{2} \operatorname{tr} \int \frac{d^{4} k}{(2 \pi)^{4}}\left(2 i g C^{\dagger}\right) \frac{i}{\not k-m} C^{T}\left(2 i g C^{\dagger}\right) \frac{i}{(\not k+\not p)-m} C^{T}
\end{aligned}
$$




$$
\begin{aligned}
& =4 g^{2} \int_{0}^{1} \int \frac{d^{4} k}{(2 \pi)^{4}} \frac{2(1-x) p^{2}+m^{2}}{\left[k^{2}-m^{2}-x(x-1) p^{2}\right]^{2}} \\
& =4 g^{2} \int_{0}^{1} \int \frac{d^{4} k}{(2 \pi)^{4}}\left[2(1-x) p^{2}+m^{2}\right] I_{0}
\end{aligned}
$$

which is only logarithmic divergent as the quadratical divergences cancel out. Using the loop regularization, the regularized $I_{0}$ has the following explicit form:

$$
I_{0}^{R}=\frac{i}{16 \pi^{2}}\left[\ln \frac{M_{c}^{2}}{\mu^{2}}-\gamma_{\omega}+y_{0}\left(\frac{\mu^{2}}{M_{c}^{2}}\right)\right]
$$

We shall adopt a subtraction scheme similar to the Minimal Subtraction scheme in dimensional regularization. For that, it is useful to introduce an arbitrary energy scale parameter $\mu_{s}$ and write $I_{0}^{R}$ as:

$$
I_{0}^{R}=\frac{i}{16 \pi^{2}} \ln \frac{M_{c}^{2}}{\mu_{s}^{2}}+\frac{i}{16 \pi^{2}}\left[\ln \frac{\mu_{s}^{2}}{\mu^{2}}-\gamma_{\omega}+y_{0}\left(\frac{\mu^{2}}{M_{c}^{2}}\right)\right]
$$

then the divergent terms proportional to $\frac{i}{16 \pi^{2}} \ln \frac{M_{c}^{2}}{\mu_{s}^{2}}$ for $M_{c} \longrightarrow \infty$ in the Feynman integral are canceled by counterterms. As such a divergent term is independent of the Feynman parameters $x$, we can integrate $x$ easily and obtain the divergent part of these diagrams:

$$
L_{\langle A A\rangle ; d i v}=\frac{i}{4 \pi^{2}} g^{2}\left(p^{2}+m^{2}\right) \ln \frac{M_{c}^{2}}{\mu_{s}^{2}}
$$

The counterterms correspondig to this divergence is:

$$
\delta L=\frac{1}{2} \delta_{A}\left(\partial_{\mu} A \partial^{\mu} A\right)-\frac{1}{2} \delta_{m_{A}} m^{2} A^{2}
$$

where

$$
\delta_{A}=-\frac{1}{4 \pi^{2}} g^{2} \ln \frac{M_{c}^{2}}{\mu_{s}^{2}} ; \quad \delta_{m_{A}}=\frac{1}{4 \pi^{2}} g^{2} \ln \frac{M_{c}^{2}}{\mu_{s}^{2}}
$$

from this we finally get:

$$
A_{\text {bare }}=\left(1-\frac{1}{8 \pi^{2}} g^{2} \ln \frac{M_{c}^{2}}{\mu_{s}^{2}}\right) A=z^{1 / 2} A ; \quad m_{\text {Abare }}=\left(1+\frac{1}{4 \pi^{2}} g^{2} \ln \frac{M_{c}^{2}}{\mu_{s}^{2}}\right) m=z^{-1} m
$$

where

$$
z=1-\frac{1}{4 \pi^{2}} g^{2} \ln \frac{M_{c}^{2}}{\mu_{s}^{2}}
$$

The calculation for $\langle B B\rangle$ is similar, which gives:

$$
B_{\text {bare }}=\left(1-\frac{1}{8 \pi^{2}} g^{2} \ln \frac{M_{c}^{2}}{\mu_{s}^{2}}\right) B=z^{1 / 2} B ; \quad m_{\text {Bbare }}=\left(1+\frac{1}{4 \pi^{2}} g^{2} \ln \frac{M_{c}^{2}}{\mu_{s}^{2}}\right) m=z^{-1} m
$$

We now turn to the calculation of $\langle\chi \chi\rangle$, from the Fig.(18) we can read directly:

$$
\begin{aligned}
L_{\langle\chi \chi\rangle}= & \int \frac{d^{4} k}{(2 \pi)^{4}}\left(2 g C^{\dagger} i\right) \frac{i}{\not k-m} C^{T}\left(2 g C^{\dagger} i\right) \frac{i}{(k-p)^{2}-m^{2}} \\
& +\int \frac{d^{4} k}{(2 \pi)^{4}}\left(2 g C^{\dagger} \gamma_{5}\right) \frac{i}{\not k-m} C^{T}\left(2 g C^{\dagger} \gamma_{5}\right) \frac{i}{(k-p)^{2}-m^{2}} \\
= & \int_{0}^{1} d x \int \frac{d^{4} k}{(2 \pi)^{4}} \frac{-8 x g^{2} C^{\dagger} \not p}{\left(k^{2}-m^{2}-x(x-1) p^{2}\right)^{2}} \\
= & -\frac{i}{4 \pi^{2}} g^{2} C^{\dagger} \not p \ln \frac{M_{c}^{2}}{\mu_{s}^{2}}+\ldots
\end{aligned}
$$


We need the following counterterms to cancel this divergence:

$$
\delta L=\frac{1}{2} \delta_{\chi} \bar{\chi} i \not \partial \chi-\frac{1}{2} \delta_{m_{\chi}} m \bar{\chi} \chi
$$

where:

$$
\delta_{\chi}=-\frac{1}{4 \pi^{2}} g^{2} \ln \frac{M_{c}^{2}}{\mu_{s}^{2}} ; \quad \delta_{m \chi}=0
$$

which indicates that the renormalization of field and mass is given by:

$$
\chi_{\text {bare }}=\left(1-\frac{1}{8 \pi^{2}} g^{2} \ln \frac{M_{c}^{2}}{\mu_{s}^{2}}\right) \chi=z^{1 / 2} \chi ; \quad m_{\chi b a r e}=\left(1+\frac{1}{4 \pi^{2}} g^{2} \ln \frac{M_{c}^{2}}{\mu_{s}^{2}}\right) m=z^{-1} m
$$

So far, we have worked out the renormalization constants for the fields $A, B$ and $\chi$ and their masses, the results agree with Eq.(6.10). Let us switch to the renormalization of coupling constant. As mentioned above, there are 7 types of vertices which should be described by only one coupling constant when supersymmetry holds. The contributions from all divergent diagrams shown in FIG 8 are found to be:

$$
\begin{aligned}
& L_{\langle A A A\rangle}=-\operatorname{tr} \int \frac{d^{4} k}{(2 \pi)^{4}}\left(2 g C^{\dagger} i\right) \frac{i}{\not p-m} C^{T}\left(2 g C^{\dagger} i\right) \frac{i}{\left(\not \not-\not p_{1}\right)-m} C^{T}\left(2 g C^{\dagger} i\right) \frac{i}{\left(\not p+\not p_{2}\right)-m} C^{T} \\
& +\frac{1}{2} \int \frac{d^{4} k}{(2 \pi)^{4}}(-6 i m g) \frac{i}{k^{2}-m^{2}} \frac{i}{\left(k+p_{1}\right)^{2}-m^{2}}\left(-12 i g^{2}\right)+\left(p_{1} \rightarrow p_{2}\right)+\left(p_{1} \rightarrow p_{3}\right) \\
& +\frac{1}{2} \int \frac{d^{4} k}{(2 \pi)^{4}}(-2 i m g) \frac{i}{k^{2}-m^{2}} \frac{i}{\left(k+p_{1}\right)^{2}-m^{2}}\left(-4 i g^{2}\right)+\left(p_{1} \rightarrow p_{2}\right)+\left(p_{1} \rightarrow p_{3}\right) \\
& =i \frac{3}{2 \pi^{2}} m g^{3} \ln \frac{M_{c}^{2}}{\mu_{s}^{2}}+\text { finite terms } \\
& L_{\langle A B B\rangle}=-\operatorname{tr} \int \frac{d^{4} k}{(2 \pi)^{4}}\left(2 g C^{\dagger} i\right) \frac{i}{\not k-m} C^{T}\left(2 g C^{\dagger} \gamma_{5}\right) \frac{i}{\left(\not \not b-\not p_{1}\right)-m} C^{T}\left(2 g C^{\dagger} \gamma_{5}\right) \frac{i}{\left(\not \not b+\not p_{2}\right)-m} C^{T} \\
& +\frac{1}{2} \int \frac{d^{4} k}{(2 \pi)^{4}}(-6 i m g) \frac{i}{k^{2}-m^{2}} \frac{i}{\left(k+p_{1}\right)^{2}-m^{2}}\left(-4 i g^{2}\right) \\
& +\frac{1}{2} \int \frac{d^{4} k}{(2 \pi)^{4}}(-2 i m g) \frac{i}{k^{2}-m^{2}} \frac{i}{\left(k+p_{1}\right)^{2}-m^{2}}\left(-12 i g^{2}\right) \\
& +\frac{1}{2} \int \frac{d^{4} k}{(2 \pi)^{4}}(-2 i m g) \frac{i}{k^{2}-m^{2}} \frac{i}{\left(k+p_{2}\right)^{2}-m^{2}}\left(-4 i g^{2}\right)+\left(p_{2} \rightarrow p_{3}\right) \\
& =i \frac{1}{2 \pi^{2}} m g^{3} \ln \frac{M_{c}^{2}}{\mu_{s}^{2}}+\text { finite terms } \\
& L_{\langle A \chi \chi\rangle}=\int \frac{d^{4} k}{(2 \pi)^{4}}\left(2 g C^{\dagger} i\right) \frac{i}{\left(\not \not+\not \not_{2}\right)-m} C^{T}\left(2 g C^{\dagger} i\right) \frac{i}{\left(\not \not+\not \phi_{1}\right)-m} C^{T}\left(2 g C^{\dagger} i\right) \frac{i}{k^{2}-m^{2}} \\
& +\int \frac{d^{4} k}{(2 \pi)^{4}}\left(2 g C^{\dagger} \gamma_{5}\right) \frac{i}{\left(\not k+\not p_{2}\right)-m} C^{T}\left(2 g C^{\dagger} i\right) \frac{i}{\left(\not k+\not p_{1}\right)-m} C^{T}\left(2 g C^{\dagger} \gamma_{5}\right) \frac{i}{k^{2}-m^{2}} \\
& =\text { finite terms } \\
& L_{\langle B \chi \chi\rangle}=\int \frac{d^{4} k}{(2 \pi)^{4}}\left(2 g C^{\dagger} i\right) \frac{i}{\left(\not k+\not p_{2}\right)-m} C^{T}\left(2 g C^{\dagger} \gamma_{5}\right) \frac{i}{\left(\not k+\not p_{1}\right)-m} C^{T}\left(2 g C^{\dagger} i\right) \frac{i}{k^{2}-m^{2}} \\
& +\int \frac{d^{4} k}{(2 \pi)^{4}}\left(2 g C^{\dagger} \gamma_{5}\right) \frac{i}{\left(\not \not+\not p_{2}\right)-m} C^{T}\left(2 g C^{\dagger} \gamma_{5}\right) \frac{i}{\left(\not k+\not p_{1}\right)-m} C^{T}\left(2 g C^{\dagger} \gamma_{5}\right) \frac{i}{k^{2}-m^{2}} \\
& =\text { finite terms }
\end{aligned}
$$




$$
\begin{aligned}
& L_{\langle A A A A\rangle}=-\operatorname{tr} \int \frac{d^{4} k}{(2 \pi)^{4}}\left(2 g C^{\dagger} i\right) \frac{i}{\not k-m} C^{T}\left(2 g C^{\dagger} i\right) \frac{i}{\left(\not k+\not p_{2}\right)-m} C^{T}\left(2 g C^{\dagger} i\right) \frac{i}{\left(\not \not b+\not \not_{2}+\not p_{3}\right)-m} \\
& C^{T}\left(2 g C^{\dagger} i\right) \frac{i}{\left(\not h+\not p_{2}+\not p_{3}+\not p_{4}\right)-m} C^{T}+\left(p_{2} \leftrightarrow p_{3}\right)+\left(p_{3} \leftrightarrow p_{4}\right) \\
& +\frac{1}{2}\left(-12 i g^{2}\right)^{2} \frac{i}{k^{2}-m^{2}} \frac{i}{\left(k+p_{1}+p_{2}\right)^{2}-m^{2}}+\left(p_{2} \rightarrow p_{3}\right)+\left(p_{2} \rightarrow p_{4}\right) \\
& +\frac{1}{2}\left(-4 i g^{2}\right)^{2} \frac{i}{k^{2}-m^{2}} \frac{i}{\left(k+p_{1}+p_{2}\right)^{2}-m^{2}}+\left(p_{2} \rightarrow p_{3}\right)+\left(p_{2} \rightarrow p_{4}\right) \\
& =i \frac{3}{\pi^{2}} g^{4} \ln \frac{M_{c}^{2}}{\mu_{s}^{2}}+\text { finite terms } \\
& L_{\langle B B B B\rangle}=-t r \int \frac{d^{4} k}{(2 \pi)^{4}}\left(2 g C^{\dagger} \gamma_{5}\right) \frac{i}{\not k-m} C^{T}\left(2 g C^{\dagger} \gamma_{5}\right) \frac{i}{\left(\not \not+\not p_{2}\right)-m} C^{T}\left(2 g C^{\dagger} \gamma_{5}\right) \frac{i}{\left(\not \not+\not p_{2}+\not p_{3}\right)-m} \\
& C^{T}\left(2 g C^{\dagger} \gamma_{5}\right) \frac{i}{\left(\not k+\not p_{2}+\not p_{3}+\not p_{4}\right)-m} C^{T}+\left(p_{2} \leftrightarrow p_{3}\right)+\left(p_{3} \leftrightarrow p_{4}\right) \\
& +\frac{1}{2}\left(-12 i g^{2}\right)^{2} \frac{i}{k^{2}-m^{2}} \frac{i}{\left(k+p_{1}+p_{2}\right)^{2}-m^{2}}+\left(p_{2} \rightarrow p_{3}\right)+\left(p_{2} \rightarrow p_{4}\right) \\
& +\frac{1}{2}\left(-4 i g^{2}\right)^{2} \frac{i}{k^{2}-m^{2}} \frac{i}{\left(k+p_{1}+p_{2}\right)^{2}-m^{2}}+\left(p_{2} \rightarrow p_{3}\right)+\left(p_{2} \rightarrow p_{4}\right) \\
& =i \frac{3}{\pi^{2}} g^{4} \ln \frac{M_{c}^{2}}{\mu_{s}^{2}}+\text { finite terms } \\
& L_{\langle A A B B\rangle}=-\operatorname{tr} \int \frac{d^{4} k}{(2 \pi)^{4}}\left(2 g C^{\dagger} i\right) \frac{i}{\not k-m} C^{T}\left(2 g C^{\dagger} i\right) \frac{i}{\left(\not k+\not p_{2}\right)-m} C^{T}\left(2 g C^{\dagger} \gamma_{5}\right) \frac{i}{\left(\not \not h+\not p_{2}+\not p_{3}\right)-m} \\
& C^{T}\left(2 g C^{\dagger} \gamma_{5}\right) \frac{i}{\left(\not h+\not p_{2}+\not p_{3}+\not p_{4}\right)-m} C^{T} \\
& -\operatorname{tr} \int \frac{d^{4} k}{(2 \pi)^{4}}\left(2 g C^{\dagger} i\right) \frac{i}{\not k-m} C^{T}\left(2 g C^{\dagger} i\right) \frac{i}{\left(\not k+\not p_{2}\right)-m} C^{T}\left(2 g C^{\dagger} \gamma_{5}\right) \frac{i}{\left(\not k+\not p_{2}+\not p_{4}\right)-m} \\
& C^{T}\left(2 g C^{\dagger} \gamma_{5}\right) \frac{i}{\left(\not k+\not p_{2}+\not p_{3}+\not p_{4}\right)-m} C^{T} \\
& -\operatorname{tr} \int \frac{d^{4} k}{(2 \pi)^{4}}\left(2 g C^{\dagger} i\right) \frac{i}{\not k-m} C^{T}\left(2 g C^{\dagger} \gamma_{5}\right) \frac{i}{\left(\not \not+\not p_{3}\right)-m} C^{T}\left(2 g C^{\dagger} i\right) \frac{i}{\left(\not \not+\not p_{3}+\not p_{2}\right)-m} \\
& C^{T}\left(2 g C^{\dagger} \gamma_{5}\right) \frac{i}{\left(\not k+\not p_{2}+\not p_{3}+\not p_{4}\right)-m} C^{T} \\
& +\frac{1}{2}\left(-4 i g^{2}\right)\left(-12 i g^{2}\right) \frac{i}{k^{2}-m^{2}} \frac{i}{\left(k+p_{1}+p_{2}\right)^{2}-m^{2}} \\
& +\frac{1}{2}\left(-12 i g^{2}\right)\left(-4 i g^{2}\right) \frac{i}{k^{2}-m^{2}} \frac{i}{\left(k+p_{1}+p_{2}\right)^{2}-m^{2}} \\
& +\left(-4 i g^{2}\right)\left(-4 i g^{2}\right)^{2} \frac{i}{k^{2}-m^{2}} \frac{i}{\left(k+p_{1}+p_{3}\right)^{2}-m^{2}}+\left(p_{3} \rightarrow p_{4}\right) \\
& =i \frac{1}{\pi^{2}} g^{4} \ln \frac{M_{c}^{2}}{\mu_{s}^{2}}+\text { finite terms }
\end{aligned}
$$

Introducing the following counterterms:

$$
\begin{aligned}
\delta L= & -\delta_{1} m g A^{3}-\delta_{2} m g A B^{2}-\delta_{3} g A \bar{\chi} \chi-\delta_{4} g B \bar{\chi} i \gamma_{5} \chi \\
& -\delta_{5} \frac{1}{2} g^{2} A^{4}-\delta_{6} \frac{1}{2} g^{2} B^{4}-\delta_{7} g^{2} A^{2} B^{2}
\end{aligned}
$$


with:

$$
\begin{aligned}
& \delta_{1}=\delta_{2}=\frac{1}{4 \pi^{2}} g^{2} \ln \frac{M_{c}^{2}}{\mu_{s}^{2}} \\
& \delta_{3}=\delta_{4}=0 \\
& \delta_{5}=\delta_{6}=\delta_{7}=\frac{1}{4 \pi^{2}} g^{2} \ln \frac{M_{c}^{2}}{\mu_{s}^{2}}
\end{aligned}
$$

It is easy to check that all the renormalized vertices lead to a single renormalization constant:

$$
g_{\text {bare }}=\left(1+\frac{3}{8 \pi^{2}} g^{2} \ln \frac{M_{c}^{2}}{\mu_{s}^{2}}\right) g=z^{-3 / 2} g
$$

This equation, together with Eq.(6.17), Eq.(6.19) and Eq.(6.23), shows that the LR method works well in the perturbative theory of massive Wess-Zumino model.

\section{CONCLUSION}

In this paper we have investigated the applicability of the recently developed Loop Regularization method in supersymmetric theories. By checking several Ward identities in various supersymmetric models, we have explicitly shown that the LR method is applicable to the supersymmetric field theories. We have also directly carried out the calculations for one-loop renormalization of massive Wess-Zumino model by using the LR method with string-mode regulators, the results are consistent with the general conclusion yielded from the supergraph technique. Once the supersymmetric extensions of the standard model could be discovered at the LHC, such a symmetrypreserving Loop Regularization method with string-mode regulators can widely be applied to the computations of various supersymmetric processes.

\section{Acknowledgments}

The authors would like to thank A.Cohen for useful discussions. This work was supported in part by the National Science Foundation of China (NSFC) under the grant \# 10821504, 10491306, and the Project of Knowledge Innovation Program (PKIP) of Chinese Academy of Science.

\section{APPENDIX A: TRANSLATIONAL INVARIANCE OF LOOP REGULARIZATION}

The verification of translational invariance in section 2 can simply be extended to the linearly and quadratically divergent integrals.

Consider firstly the quadratically divergent integral

$$
L_{2}=\int \frac{d^{4} k}{\left[(k-x p)^{2}+M^{2}\right]}
$$

by rewriting the momentum factor $(k-x p)^{2}$ into $(k-x p)^{2}=k^{2}-2 x p . k+x^{2} p^{2}$, then replacing $k^{2}$ by $k^{2}+M_{l}^{2}$, one has

$$
(k-x p)^{2} \rightarrow k^{2}+M_{l}^{2}-2 x p . k+x^{2} p^{2}=(k-x p)^{2}+M_{l}^{2}
$$


Thus the proof in the manuscript for the scalar type logarithmic loop integration can be easily extended to the scalar type quadratically divergent ILIs, namely

$$
L_{2} \rightarrow L_{2}^{R}=\lim _{N, M_{l}^{2}} \sum_{l=0}^{N} c_{l}^{N} \int \frac{d^{4} k}{\left[(k-x p)^{2}+M_{l}^{2}\right]}
$$

The regularized ILIs $L_{2}^{R}$ is well-defined and allows us to shift the momentum, we then have

$$
L_{2}^{R}=\lim _{N, M_{l}^{2}} \sum_{l=0}^{N} c_{l}^{N} \int \frac{d^{4} k}{\left[(k-x p)^{2}+M_{l}^{2}\right]}=\lim _{N, M_{l}^{2}} \sum_{l=0}^{N} c_{l}^{N} \int \frac{d^{4} k}{\left[k^{2}+M_{l}^{2}\right]}=I_{2}^{R}
$$

Actually, it is this translational invariance which allows us to clarify the ambiguity caused by the linear divergent in evaluating the triangle anomaly and CPT/Lorentz violating Chern-Simons term, which was shown in ref. [14]. To be more clear here, we demonstrate it as follows.

Let's first present J. Jauch and F. Rohrlich's discussion on the logarithmically divergent integrals 23$]$. Considering the following integral,

$$
L_{0}=\int \frac{d^{4} k}{\left[(k-p)^{2}+M^{2}\right]^{2}}
$$

and making use of the identity,

$$
\frac{1}{\alpha^{n}}-\frac{1}{\beta^{n}}=-\int_{0}^{1} \frac{n(\alpha-\beta) d z}{[(\alpha-\beta) z+\beta]^{n+1}}
$$

for $n=2$, we can rewrite the above integral as follows

$$
L_{0}=\int \frac{d^{4} k}{\left(k^{2}+M^{2}\right)^{2}}-2 \int d^{4} k \int_{0}^{1} \frac{\left(p^{2}-2 p \cdot k\right) d z}{\left[k^{2}+M^{2}+\left(p^{2}-2 p \cdot k\right) z\right]^{3}} \equiv I_{0}+L_{c}
$$

The second term $L_{c}$ of the right-hand side is convergent, so we can safely shift the origin of $k$

$$
k_{\mu} \rightarrow k_{\mu}+p_{\mu} z
$$

and the second term reads

$$
L_{c}=-2 \int_{0}^{1} d z \int \frac{p^{2}(1-2 z)-2 p \cdot k}{\left[k^{2}+M^{2}+p^{2} z(1-z)\right]^{3}} d^{4} k
$$

The term in the numerator which is odd in $\mathrm{k}$ will vanish. Using the identity

$$
\int \frac{\left(k^{2}\right)^{m-2} d^{4} k}{\left(k^{2}+M^{2}\right)^{n}}=\frac{i \pi^{2}}{\left(M^{2}\right)^{n-m}} B(m, n-m)
$$

where $B(m, n-m)=\Gamma(m) \Gamma(n-m) / \Gamma(n)$ and $n>m>0$ is the condition of convergence. So the second term in eq(A7) now goes as

$$
L_{c}=-2 \frac{i \pi^{2}}{2} \int_{0}^{1} d z \frac{p^{2}(1-2 z)}{M^{2}+p^{2} z(1-z)}=-\left.i \pi^{2} \ln \left[M^{2}+p^{2} z(1-z)\right]\right|_{0} ^{1}=0
$$

Therefore, for the logarithmic divergent integral, we arrive at the following identity

$$
L_{0}=\int \frac{d^{4} k}{\left[(k-p)^{2}+M^{2}\right]^{2}}=\int \frac{d^{4} k}{\left[k^{2}+M^{2}\right]^{2}}=I_{0}
$$


which is independent of the regularization.

Nevertheless, if firstly applying the Loop Regularization prescription and then shifting the momentum, the corresponding relation becomes a straightforward consequence

$$
L_{0} \rightarrow L_{0}^{R}=\lim _{N, M_{l}^{2}} \sum_{l=0}^{N} c_{l}^{N} \int \frac{d^{4} k}{\left[(k-p)^{2}+M_{l}^{2}\right]^{2}}=\lim _{N, M_{l}^{2}} \sum_{l=0}^{N} c_{l}^{N} \int \frac{d^{4} k}{\left[k^{2}+M_{l}^{2}\right]^{2}}=I_{0}^{R}
$$

Let us now consider the linear divergent integral. When using the identity eq. (A6), a similar proof can be carried out and shows that a shift of $k$ in a linearly divergent integral will result in a finite additive constant

$$
L_{1, \mu}=\int \frac{k^{\mu} d^{4} k}{\left[(k-p)^{2}+M^{2}\right]^{2}}=\int \frac{(k+p)^{\mu} d^{4} k}{\left[k^{2}+M^{2}\right]^{2}}-\frac{i \pi^{2}}{2} p^{\mu} \equiv I_{1 \mu}+p_{\mu} I_{0}+L_{c \mu}
$$

which has been shown to cause an ambiguity in evaluating the chiral anomaly if the regularization schemes are not applied appropriately [14]. This is because the results may depend on the procedure of applying the regularization schemes before or after using the identity eq. (A6).

To be safe, we shall apply LR prescription before shifting the momentum, it then leads to the following result

$$
\begin{aligned}
L_{1, \mu} & \rightarrow L_{1, \mu}^{R}=\lim _{N, M_{l}^{2}} \sum_{l=0}^{N} c_{l}^{N} \int \frac{k^{\mu} d^{4} k}{\left[(k-p)^{2}+M_{l}^{2}\right]^{2}} \\
& =\lim _{N, M_{l}^{2}} \sum_{l=0}^{N} c_{l}^{N} \int \frac{(k+p)^{\mu} d^{4} k}{\left[k^{2}+M_{l}^{2}\right]^{2}}=\lim _{N, M_{l}^{2}} \sum_{l=0}^{N} c_{l}^{N} \int \frac{p^{\mu} d^{4} k}{\left[k^{2}+M_{l}^{2}\right]^{2}}=p_{\mu} I_{0}^{R}
\end{aligned}
$$

where we have shifted the momentum for the well-defined regularized integral but without using the above identity.

On the other hand, when applying LR prescription before shifting the momentum, but using the identity presented above for the integration, we then arrive at the following expression

$$
\begin{aligned}
L_{1, \mu} \rightarrow L_{1, \mu}^{R} & =\lim _{N, M_{l}^{2}} \sum_{l=0}^{N} c_{l}^{N} \int \frac{k^{\mu} d^{4} k}{\left[(k-p)^{2}+M_{l}^{2}\right]^{2}} \\
& =\lim _{N, M_{l}^{2}} \sum_{l=0}^{N} c_{l}^{N} \int \frac{(k+p)^{\mu} d^{4} k}{\left[k^{2}+M_{l}^{2}\right]^{2}}-\frac{i \pi^{2}}{2} p^{\mu} \lim _{N, M_{l}^{2}} \sum_{l=0}^{N} c_{l}^{N}
\end{aligned}
$$

The second term of the right-hand side actually vanishes due to the following conditions for the coefficients in LR

$$
\lim _{N, M_{l}^{2}} \sum_{l=0}^{N} c_{l}^{N}\left(M_{l}^{2}\right)^{n}=0 \quad(n=0,1, \cdots)
$$

thus we finally yield the following relation

$$
\begin{aligned}
L_{1, \mu}^{R} & =\lim _{N, M_{l}^{2}} \sum_{l=0}^{N} c_{l}^{N} \int \frac{k^{\mu} d^{4} k}{\left[(k-p)^{2}+M_{l}^{2}\right]^{2}}=\lim _{N, M_{l}^{2}} \sum_{l=0}^{N} c_{l}^{N} \int \frac{(k+p)^{\mu} d^{4} k}{\left[k^{2}+M_{l}^{2}\right]^{2}} \\
& =\lim _{N, M_{l}^{2}} \sum_{l=0}^{N} c_{l}^{N} \int \frac{p^{\mu} d^{4} k}{\left[k^{2}+M_{l}^{2}\right]^{2}}=p_{\mu} I_{0}^{R}
\end{aligned}
$$

which just shows that in the LR method the translation of momentum can safely be made for a linearly divergent integral. 
Now we turn to the quadratically divergent integral,

$$
L_{2}=\int \frac{d^{4} k}{\left[(k-p)^{2}+M^{2}\right]}
$$

which can be rewritten as follows when using the previous identity

$$
L_{2}=\int \frac{d^{4} k}{\left(k^{2}+M^{2}\right)}-2 \int d^{4} k \int_{0}^{1} \frac{\left(p^{2}-2 p \cdot k\right) d z}{\left[k^{2}+M^{2}+\left(p^{2}-2 p \cdot k\right) z\right]^{2}} \equiv I_{2}+L_{2 c}
$$

Since the second term involves only linear and logarithmical divergences, we can then use the previous identities for those integrals when shifting the origin of $k$, and get the following result with a finite additive constant

$$
\begin{aligned}
& L_{2 c}=-2 \int d^{4} k \int_{0}^{1} \frac{\left(p^{2}-2 p \cdot k\right) d z}{\left[k^{2}+M^{2}+\left(p^{2}-2 p \cdot k\right) z\right]^{2}} \\
= & -2 \int_{0}^{1} d z \int \frac{p^{2} d^{4} k}{\left[k^{2}+M^{2}+p^{2} z(1-z)\right]^{2}} \\
+ & 2 \int_{0}^{1} d z \int \frac{2 p \cdot(k+x p) d^{4} k}{\left[k^{2}+M^{2}+p^{2} z(1-z)\right]^{2}}-i \pi^{2} p^{2}
\end{aligned}
$$

The term which is odd in $k$ does not contribute, and two integrals of the right-hand side cancel each other due to the relation

$$
\int_{0}^{1} d z \int \frac{z p^{2} d^{4} k}{\left[k^{2}+M^{2}+p^{2} z(1-z)\right]^{2}}=\frac{1}{2} \int_{0}^{1} d z \int \frac{p^{2} d^{4} k}{\left[k^{2}+M^{2}+p^{2} z(1-z)\right]^{2}}
$$

Thus we arrive at the following identity

$$
L_{2}=\int \frac{d^{4} k}{\left[(k-p)^{2}+M^{2}\right]}=\int \frac{d^{4} k}{\left[k^{2}+M^{2}\right]}-i \pi^{2} p^{2} \equiv I_{2}+L_{2 c}
$$

Just like the discussion in linearly divergent integral, by applying the LR prescription before shifting momentum, we have

$$
L_{2} \rightarrow L_{2}^{R}=\lim _{N, M_{l}^{2}} \sum_{l=0}^{N} c_{l}^{N} \int \frac{d^{4} k}{\left[(k-p)^{2}+M_{l}^{2}\right]}=\lim _{N, M_{l}^{2}} \sum_{l=0}^{N} c_{l}^{N} \int \frac{d^{4} k}{\left[k^{2}+M_{l}^{2}\right]}=I_{2}^{R}
$$

where the shift of momentum has been made for the regularized $L_{2}^{R}$. On the other hand, again applying the LR prescript before shifting momentum, but using the identity obtained above, we arrive at the following expression

$$
\begin{aligned}
L_{2} \rightarrow L_{2}^{R} & =\lim _{N, M_{l}^{2}} \sum_{l=0}^{N} c_{l}^{N} \int \frac{d^{4} k}{\left[(k-p)^{2}+M_{l}^{2}\right]} \\
& =\lim _{N, M_{l}^{2}} \sum_{l=0}^{N} c_{l}^{N} \int \frac{d^{4} k}{\left[k^{2}+M_{l}^{2}\right]}-i \pi^{2} p^{2} \lim _{N, M_{l}^{2}} \sum_{l=0}^{N} c_{l}^{N}
\end{aligned}
$$

accordingly, because of the vanish of the second term in the right-hand side, we obtain the same regularized result

$$
L_{2}^{R}=\lim _{N, M_{l}^{2}} \sum_{l=0}^{N} c_{l}^{N} \int \frac{d^{4} k}{\left[(k-p)^{2}+M_{l}^{2}\right]}=\lim _{N, M_{l}^{2}} \sum_{l=0}^{N} c_{l}^{N} \int \frac{d^{4} k}{\left[k^{2}+M_{l}^{2}\right]}=I_{2}^{R}
$$

So far we have demonstrated that Loop Regularization can preserve translational invariance not only in logarithmically, but also in linearly and quadratically divergent integral. 


\section{APPENDIX B: DERIVATION OF MAJORANA FEYNMAN RULES}

Here we are going to present a simple and definite derivation of Majorana Feynman rules which are useful for our calculations in this paper. We will begin with the quantization of free Majorana fermion, and figure out the difficulties of formulating the Majorana Feynman rules, then provide a consistent prescription. The unusual Majorana Feynman rules are result from the Majorana fermion self-conjugacy. Though the two-components formulation of Majorana field is more fundamental, it is still very useful to work in four-components formalism because the $\gamma$ matrices is more convenient for practical calculations.

The Majorana fermion field $\chi$ is quantized by stipulating the following equal-time anticommutators:

$$
\begin{aligned}
& \left\{\chi_{\alpha}(\mathbf{x}), \chi_{\beta}^{\dagger}(\mathbf{y})\right\}=\delta_{\alpha \beta} \delta^{3}(\mathbf{x}-\mathbf{y}) \\
& \left\{\chi_{\alpha}(\mathbf{x}), \chi_{\beta}(\mathbf{y})\right\}=\left\{\chi_{\alpha}^{\dagger}(\mathbf{x}), \chi_{\beta}^{\dagger}(\mathbf{y})\right\}=0
\end{aligned}
$$

The plane wave decomposition of $\chi$ is not obvious. In two-components formalism the difficulty behaves as that the equation of motion (EOM) is no longer a linear equation since the EOM connects $\chi$ to its complex conjugation. In four-components formalism the difficulty lies in the Majorana condition: $\chi=\chi^{c}=C \bar{\chi}^{T}$. But if we use the spinors $u$ and $v$ which satisfy $u_{\mathbf{k}, s}=C \bar{v}_{\mathbf{k}, s}^{T}$ and $v_{\mathbf{k}, s}=C \bar{u}_{\mathbf{k}, s}^{T}$, then $\chi$ can be expanded as:

$$
\chi=\int \frac{d^{3} k}{(2 \pi)^{3}} \frac{1}{2 E_{\mathbf{k}}} \sum_{s}\left[c_{\mathbf{k}, s} u_{\mathbf{k}, s} e^{-i k x}+c_{\mathbf{k}, s}^{\dagger} v_{\mathbf{k}, s} e^{i k x}\right]
$$

here $c$ and $c^{\dagger}$ are the annihilation and creation operators of Majorana fermions. For Majorana fields, we still have:

$$
\left\langle 0\left|T \chi_{\alpha}(x) \bar{\chi}_{\beta}(y)\right| 0\right\rangle=\int \frac{d^{4} k}{(2 \pi)^{4}} e^{-i k(x-y)}\left(\frac{i}{\not k-m}\right)_{\alpha \beta}=S_{F \alpha \beta}(x-y)
$$

Note that because of the Majorana condition $\chi=C \bar{\chi}^{T}$ and $\bar{\chi}=\chi^{T} C,\left\langle 0\left|T \chi_{\alpha}(x) \chi_{\beta}(y)\right| 0\right\rangle$ and $\left\langle 0\left|T \bar{\chi}_{\alpha}(x) \bar{\chi}_{\beta}(y)\right| 0\right\rangle$ do not vanish. It is easy to show that:

$$
\begin{aligned}
& \left\langle 0\left|T \chi_{\alpha}(x) \chi_{\beta}(y)\right| 0\right\rangle=S_{F \alpha \gamma}(x-y) C_{\gamma \beta}^{T} \\
& \left\langle 0\left|T \bar{\chi}_{\alpha}(x) \bar{\chi}_{\beta}(y)\right| 0\right\rangle=C_{\alpha \gamma}^{T} S_{F \gamma \beta}(x-y)
\end{aligned}
$$

The explicit expressions of $u_{\mathbf{k}_{, s}}$ and $v_{\mathbf{k}, s}$ as well as the spin-sum identities can be found in [22], we list the results here:

$$
\begin{gathered}
u_{\mathbf{k}, s}=\left(\begin{array}{c}
\sqrt{k \cdot \sigma} \zeta_{s} \\
\sqrt{k \cdot \bar{\sigma}} \zeta_{s}
\end{array}\right), \quad \bar{u}_{\mathbf{k}, s}=\left(\zeta_{s}^{\dagger} \sqrt{k \cdot \bar{\sigma}}, \zeta_{s}^{\dagger} \sqrt{k \cdot \sigma}\right) \\
v_{\mathbf{k}, s}=\left(\begin{array}{c}
2 s \sqrt{k \cdot \sigma} \zeta_{-s} \\
-2 s \sqrt{k \cdot \bar{\sigma}} \zeta_{-s}
\end{array}\right), \bar{v}_{\mathbf{k}, s}=\left(-2 s \zeta_{s}^{\dagger} \sqrt{k \cdot \bar{\sigma}}, 2 s \zeta_{s}^{\dagger} \sqrt{k \cdot \sigma}\right)
\end{gathered}
$$

and $\zeta_{ \pm 1 / 2}$ are defined as below (here $\theta$ is the polar angle of $\mathbf{k}$, and $\phi$ is the azimuthal angle of $\mathbf{k}$.):

$$
\zeta_{1 / 2}(\mathbf{k})=\left(\begin{array}{c}
\cos \frac{\theta}{2} \\
e^{i \phi} \sin \frac{\theta}{2}
\end{array}\right), \quad \zeta_{-1 / 2}(\mathbf{k})=\left(\begin{array}{c}
-e^{-i \phi} \sin \frac{\theta}{2} \\
\cos \frac{\theta}{2}
\end{array}\right)
$$


The spin-sum identities are:

$$
\begin{aligned}
& \sum_{s} u_{\mathbf{k}, s} \bar{u}_{\mathbf{k}, s}=\not \not+m \\
& \sum_{s} v_{\mathbf{k}, s} \bar{v}_{\mathbf{k}, s}=\not \not-m \\
& \sum_{s} u_{\mathbf{k}, s} v_{\mathbf{k}, s}^{T}=(\not \not+m) C^{T} \\
& \sum_{s} v_{\mathbf{k}, s} u_{\mathbf{k}, s}^{T}=(\not \not-m) C^{T} \\
& \sum_{s} \bar{u}_{\mathbf{k}, s}^{T} \bar{v}_{\mathbf{k}, s}=C^{\dagger}(\not \not-m) \\
& \sum_{s} \bar{v}_{\mathbf{k}, s}^{T} \bar{u}_{\mathbf{k}, s}=C^{\dagger}(\not \not+m)
\end{aligned}
$$

Before starting the derivation of Majorana Feynman rules we may briefly review the derivation for the usual Dirac fermions. The argument below follows the one in [24]. The calculation of a typical scattering matrix element corresponds to the evaluation of the following expression:

$$
\left\langle 0\left|b_{1} \ldots b_{m} d_{1} \ldots d_{n} T\left[\left(\bar{\psi}\left(x_{1}\right) \Gamma \psi\left(x_{1}\right)\right) \ldots\left(\bar{\psi}\left(x_{l}\right) \Gamma \psi\left(x_{l}\right)\right)\right] b_{1}^{\dagger} \ldots b_{p}^{\dagger} d_{1}^{\dagger} \ldots d_{q}^{\dagger}\right| 0\right\rangle
$$

Firstly, we should rearrange the interaction terms to make them following the order of contractions. Since only one type of contraction $\langle\psi \bar{\psi}\rangle$ exists for Dirac fermion, the internal propagator reads: $\langle\psi \bar{\psi}\rangle=S_{F}(p)$, here the fermion charge and the momentum flows are well defined from $\bar{\psi}$ to $\psi$, the Feynman rule for vertex directly reads as $i \Gamma$. For Dirac fermion, the fermion charge flow (in fact this is also the momentum flow) of internal popagator forms a continuous flow, when writing down the analytic expression one should first do it oppositing to the continuous flow. The most important step is to determine the Relative Sign of Interfering Feynman graphs (RSIF). There are in general three types of commutations which can contribute to the RSIF. Firstly, when reordering $b_{i}, d_{i}, b_{i}^{\dagger}$ and $d_{i}^{\dagger}$ to put them in the appropriate places of Wick contractions, it causes a factor $(-1)^{P}$. Here $P$ is the parity of the permutation of the annihilation and creation operators. This factor can be read from the order of external spinors in the analytic expression with respect to the given reference order. Secondly, for a closed fermion loop, one needs to exchange the first and the last field operator in the fermion chain, which gives a factor $(-1)^{L}$, where $L$ is the number of fermion loops. Finally, since $d_{i}^{\dagger}$ must contract with $\bar{\psi}$ and $d_{i}$ must contract with $\psi$, one needs to move the creation operator $d_{i}$ to the beginning of Wick contraction and move the annihilation operator $d_{i}^{\dagger}$ to the end, which leads to a factor $(-1)^{V}$ with $V$ being the total number of spinors $v$ and $\bar{v}$. Since $V$ is universal for all graphs of a given process, this factor can therefore be omitted.

We now trun to investigate the Majorana fermion case. Firstly, we consider the situation that there are no Dirac fermions but only Majorana fermions. As mentioned above, all possible contractions between $\chi$ and/or $\bar{\chi}$ do not vanish now. In this case, after rearranging the interaction terms to perform Wick contraction for operators one by one, we need to consider four types of Majorana propagators, i.e. $\langle\chi \chi\rangle,\langle\chi \bar{\chi}\rangle,\langle\bar{\chi} \chi\rangle$ and $\langle\bar{\chi} \bar{\chi}\rangle$. More seriously, the propagators depend on the sign of its momentum $p$, but now we can not define the orientation from $\bar{\chi}$ to $\chi$ as the arrow of momentum. That means we need to find out a new method to resign the arrow of momentum. For the Feynman rule of vertex, it raises a new ambiguity. For instance, when contracting an interaction Lagrangian $\bar{\chi} \Gamma \chi$ in the time-order product, one can contract the operator $\bar{\chi}$ with one field operator lies on the left of this vertex and contract $\chi$ with another lies on the right, or one can also contract $\chi$ with one field operator lies on the left and contract $\bar{\chi}$ with another lies on the right. In the later case an additional $(-1)$ will emerge. Previous discussions [25, 26] for the Majorana Feynman rules follows this analysis and try to reduce the number of propagators and 
vertices, while the resulting consequences are still too obscure and not easy to use. In ref[24], the author introduced the charge-conjugate fields $\psi^{c}$ and $\bar{\psi}^{c}$ to Feynman rules and tried to give a uniform description of Dirac and Majorana field. Here we shall provide an alternative and simple description.

Firstly, we may eliminate $\bar{\chi}$ from the interaction Lagrangian by using the Majorana condition $\bar{\chi}=-\chi^{T} C^{\dagger}$, so that only one type of propagator $\langle\chi \chi\rangle$ remains. We then use a line without arrow to represent a Majorana propagator. Since Majorana fermions can not carry any charge, this representation is natural. In the momentum space, the Feynman rule for Majorana propagator is $\frac{i}{k-m} C^{T}$. To obtain the Feynman rule of vertex, we may rewrite $\bar{\chi} \Gamma \chi$ as:

$$
\begin{aligned}
\bar{\chi}_{\alpha} \Gamma_{\alpha \beta} \chi_{\beta} & =\chi_{\alpha}\left(-C_{\alpha \rho}^{\dagger} \Gamma_{\rho \beta}\right) \chi_{\beta}=-\chi_{\beta}\left(\Gamma_{\beta \rho}^{T} C_{\rho \alpha}^{\dagger}\right) \chi_{\alpha}=\frac{1}{2} \chi_{\alpha}\left(-C^{\dagger} \Gamma-\Gamma^{T} C^{\dagger}\right)_{\alpha \beta} \chi_{\beta} \\
& =\frac{1}{2} \chi_{\alpha} \Gamma_{\alpha \beta}^{\prime} \chi_{\beta}
\end{aligned}
$$

with:

$$
\Gamma^{\prime}=-C^{\dagger} \Gamma-\Gamma^{T} C^{\dagger}=-\Gamma^{\prime T}
$$

Now the ambiguity mentioned about disappears as $\Gamma^{\prime}$ is antisymmetric. The Feynman rule for vertex simply becomes: $i \Gamma^{\prime}$. One can treat the Majorana fermions just like a real scalar boson to obtain the correct symmetric factor of a given graph.

Next, we should determine the direction of momentum in Majorana propagators. Remember that generally a factor $e^{-i k x}$ means momentum $k$ flows in the point $x$ and $e^{i k x}$ means momentum $\mathrm{k}$ flows out the point $x$. Every contraction between two field operators $O(x), O(y)$ can always be written in the form: $\langle O(x) O(y)\rangle=\int \frac{d^{4} k}{(2 \pi)^{4}} e^{-i k(x-y)} S(k)$, for example in our case:

$$
\left\langle 0\left|T \chi_{\alpha}(x) \chi_{\beta}(y)\right| 0\right\rangle=\int \frac{d^{4} k}{(2 \pi)^{4}} e^{-i k(x-y)}\left(\frac{i}{\not k-m} C^{T}\right)_{\alpha \beta}
$$

which indicates that the direction of momentum flow is always opposite to the direction of contraction for a propagator, and in a fermion chain the momentum flows of propagators form a continuous flow its direction is opposite to the direction of contractions. In [24] such a folw was called as 'fermion flow', here we may, more precisely, call it as 'fermion momentum flow'. This comes to the conclusion: for each fermion chain we fix an arbitrary orientation (fermion momentum flow), the momentums of all fermion propagators follow this orientation, and we should write down the Feynman rules proceeding opposite to the chosen orientation.

Finally, to complete the Majorana Feynman rules, it needs to give the rules of external fermion lines and determine the RSIF. The rules of external fermion lines can easily be obtained from the plan-wave decomposition of $\chi$, see Eqn.(B2). Since

$$
\begin{aligned}
\langle 0| c_{\mathbf{k}, s} \chi_{\alpha}(x) & \longrightarrow v_{\alpha \mathbf{k}, s} e^{i k x} \\
\chi_{\alpha}(x) c_{\mathbf{k}, s}^{\dagger}|0\rangle & \longrightarrow u_{\alpha \mathbf{k}, s} e^{-i k x}
\end{aligned}
$$

which implies that the creation of a Majorana fermion corresponds to a spinor $v_{\alpha \mathbf{k}, s}$ with momentum $k$ flow out, and the annihilation of a Majorana fermion corresponds to a spinor $u_{\alpha \mathbf{k}, s}$ with momentum $k$ flow in. If the spinor locates at the beginning of contraction, we should write it as a row vector say a $u^{T}$ or $v^{T}$. Now we can give a prescription to fix RSIF. Factor $(-1)^{P}$ can be got from the permutation parity of the spinors in the obtained analytical expression with respect to some reference order. Factor $(-1)^{L}$ can be got from the number of closed fermion loops. Factor 
$(-1)^{V}$ now is a little different from which in Dirac field theory. Since moving any one creation operator arising from the initial state to the beginning of contraction will contribute a factor -1 , and moving any one annihilation operator arising from the final state to the end of contraction which also contribute a factor -1 , it seems that we should count the total number of such operation. Suppose that there are ' $a$ ' fermions in the initial sate and ' $b$ ' fermions in the final state, and we must move $i$ th fermion creation operators to the beginning and $j$ th fermion annihilation operators to the end, then we have $a-i+j=b+i-j$, i.e. $|i-j|=\frac{1}{2}|a-b|$. Namely, $V=\frac{1}{2}|a-b|$. Since $a$ and $b$ is universal for all graphs of a process, we can always omit $(-1)^{V}$ all the time.

Let us consider the situation that a Majorana fermion $\chi$ couples to a Dirac fermion $\psi$, the interaction Lagrangian contains the following terms:

$$
\bar{\chi} \Gamma \psi+\bar{\psi} \bar{\Gamma} \chi \quad\left(\text { where }: \bar{\Gamma}=\gamma^{0} \Gamma^{\dagger} \gamma^{0}\right)
$$

When keeping a continuous "fermion momentum flow" for a fermion internal line, we then need to consider two types of Dirac propagators: $\langle\psi \bar{\psi}\rangle$ and $\langle\bar{\psi} \psi\rangle$ which have the following explicit forms:

$$
\begin{aligned}
\left\langle 0\left|T \psi_{\alpha}(x) \bar{\psi}_{\beta}(y)\right| 0\right\rangle & =\int \frac{d^{4} k}{(2 \pi)^{4}} e^{-i k(x-y)}\left(\frac{i}{\not k-m}\right)_{\alpha \beta} \\
\left\langle 0\left|T \bar{\psi}_{\alpha}(x) \psi_{\beta}(y)\right| 0\right\rangle & =\int \frac{d^{4} k}{(2 \pi)^{4}} e^{-i k(x-y)}\left[\left(\frac{i}{\not k+m}\right)^{T}\right]_{\alpha \beta}
\end{aligned}
$$

We then need to use a line with arrow to represent the Dirac propagator, the arrow reflects the flow of charge which flows out of $\bar{\psi}$ and into $\psi$. If the direction of charge flow coincide with the direction of the "fermion momentum flow", we should use $\langle\psi \bar{\psi}\rangle=\frac{i}{k-m}$, otherwise we should use $\langle\bar{\psi} \psi\rangle=\left(\frac{i}{k+m}\right)^{T}$.

The Feynman rules for vertexes are also doubled. For the Dirac-Dirac interaction, one has:

$$
\bar{\psi}_{\alpha} \Gamma_{\alpha \beta} \psi_{\beta}=\psi_{\alpha}\left(-\Gamma^{T}\right)_{\alpha \beta} \bar{\psi}_{\beta}
$$

If the direction of charge flow coincide with the direction of the momentum flow, we should use $i \Gamma$, otherwise we should use $-i \Gamma^{T}$. the vertexes rules of Majorana-Dirac interaction can be derived similarly from the identities:

$$
\begin{aligned}
\bar{\chi}_{\alpha} \Gamma_{\alpha \beta} \psi_{\beta} & =\chi_{\alpha}\left(-C^{\dagger} \Gamma\right)_{\alpha \beta} \psi_{\beta}=\psi_{\alpha}\left(-\Gamma^{T} C^{\dagger}\right)_{\alpha \beta} \chi_{\beta} \\
\bar{\psi}_{\alpha} \bar{\Gamma}_{\alpha \beta} \chi_{\beta} & =\chi_{\alpha}\left(-\bar{\Gamma}^{T}\right)_{\alpha \beta} \bar{\psi}_{\beta}
\end{aligned}
$$

The RSIF can be determined by using the same method as we mentioned above.

With the above considerations, we can summarize our Feynman rules. The solid lines are still used to denote the fermions. Dirac fermions lines carry arrows which reflect the direction of charge flow, Majorana lines do not carry arrows. We may write down Feynman amplitudes according to the following steps:

1. Draw all topologically distinctive, connect Feynman diagrams for a given process.

2. Fix an arbitrary direction for each fermion chain. This is the direction of "fermion momentum flow", which means that the momentum of every internal fermion line should follow this direction. We should write down the Dirac matrices proceeding opposite to the chosen direction through the chain.

3. For the external fermion lines, the rules are shown in FIG 9 .

If the spinors locates at the beginning(end) of contraction, we should add a superscript $T$ appropriately to write them as row(column) vectors.

4. For the fermion propagators, the rules are shown in FIG.10. 


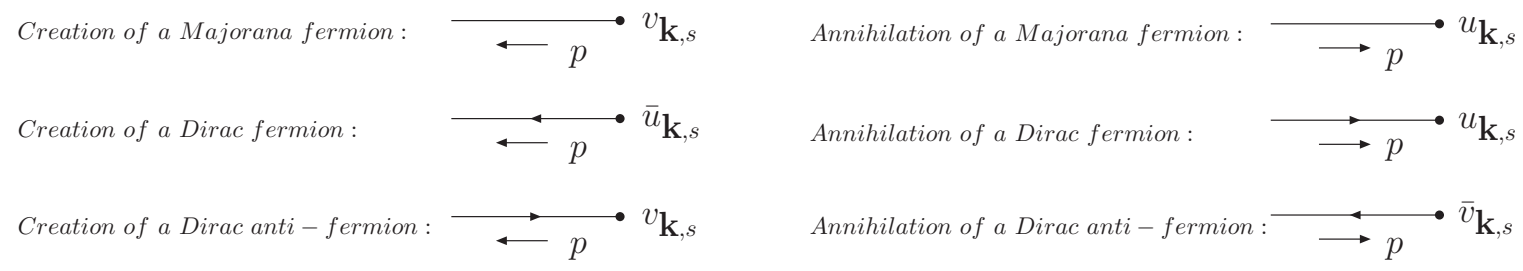

FIG. 9: Feynman rules for external lines

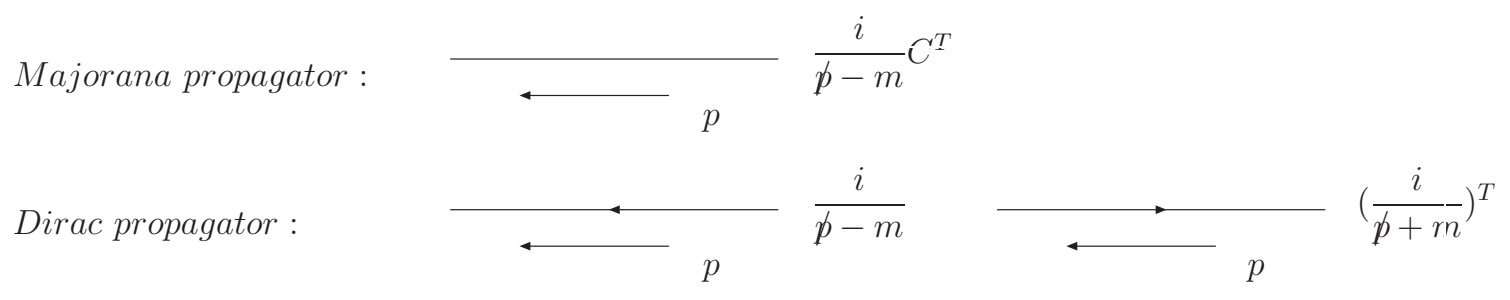

FIG. 10: Feynman rules for propagators

5. For the general fermion interactions $\bar{\chi} \Gamma_{1} \chi, \bar{\psi} \Gamma_{2} \psi, \bar{\chi} \Gamma_{3} \psi+\bar{\psi} \bar{\Gamma}_{3} \chi$, where $\bar{\Gamma}_{3}=\gamma^{0} \Gamma_{3}^{\dagger} \gamma^{0}$, the Feynman rules are shown in FIG 11 respectively.

6. To determine the RSIF. For each diagram, multipling by a factor $(-1)$ for each closed fermion loop, and multipling by the permutation parity of the spinors in the obtained analytical expression with respect to some reference order.

7. Multipling a symmetry factor $S^{-1}$ for each diagram. The Majorana fermions may be treated just as real scalar fields to obtain the symmetry factor.

$$
S=g \prod_{n=2,3, \ldots} 2^{\beta}(n !)^{\alpha_{n}}
$$

where $\alpha_{n}$ is the number of pairs of vertices connected by $n$ identical self-conjugate lines, $\beta$ is the number of lines connecting a vertex with itself, and $g$ is the number of permutations of vertices which leave the diagram unchanged with fixed external lines. 

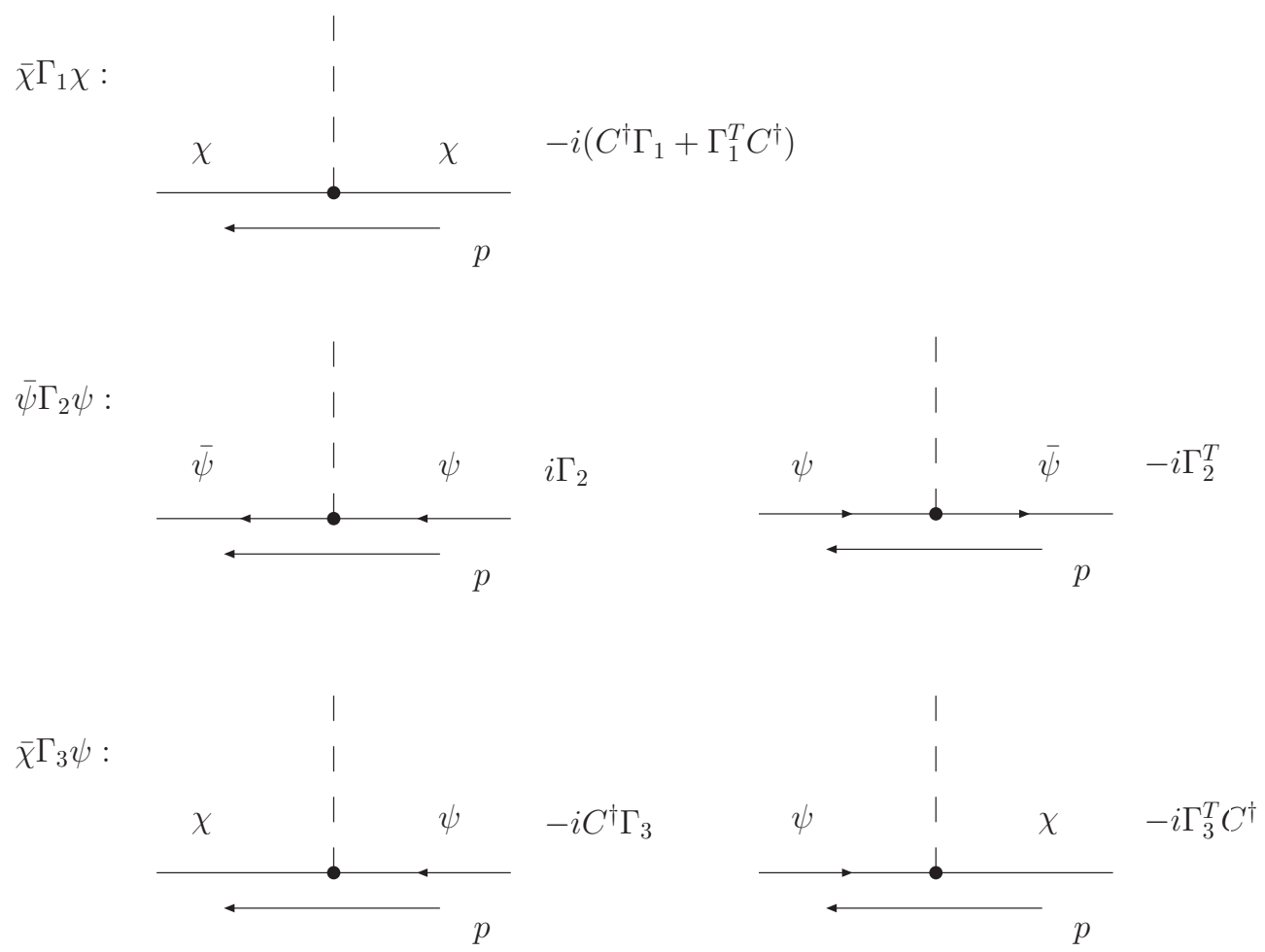

$\bar{\psi} \bar{\Gamma}_{3} \chi:$
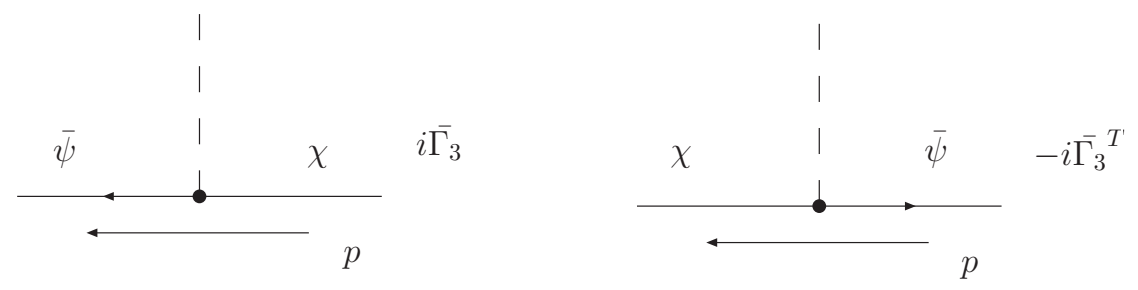

FIG. 11: Feynman rules for vertexes

APPENDIX C: FEYNMAN RULES OF MASSIVE WESS-ZUMINO MODEL

We present all the Feynman rules of this model in FIG,12. 


$$
\varlimsup_{A(B)}^{m_{A(B)}} \frac{i}{k^{2}-m^{2}} \quad \chi_{\chi} \frac{i}{p-m} C^{T}
$$
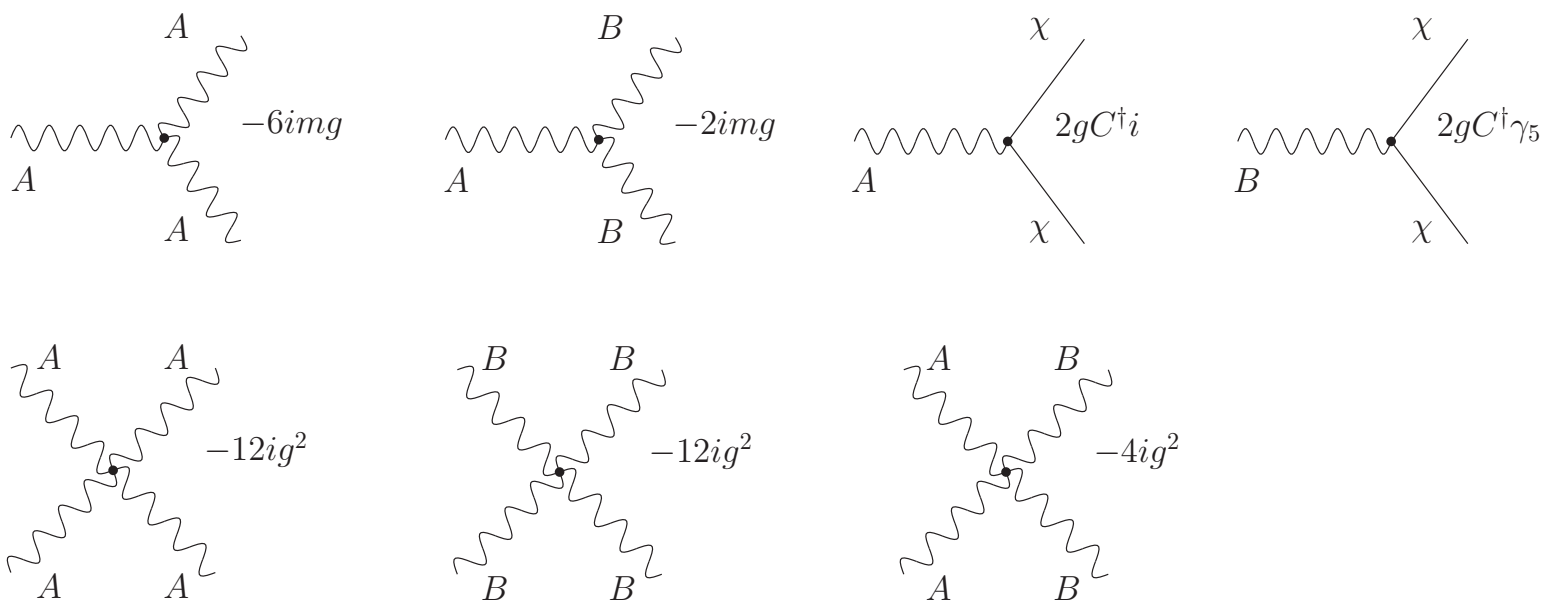

FIG. 12: Feynman rules of massive Wess-Zumino model

[1] Yu. A. Golfand and E. P. Likhtman, JETP Lett. 13, 323 (1971) [Pisma Zh. Eksp. Teor. Fiz. 13, 452 (1971)].

J. Wess and B. Zumino, Nucl. Phys. B 70, 39 (1974).

[2] N. Maggiore, O. Piguet and S. Wolf, Nucl. Phys. B 458, 403 (1996) [Erratum-ibid. B 469, 513 (1996)] arXiv:hep-th/9507045.

N. Maggiore, O. Piguet and S. Wolf, Nucl. Phys. B 476, 329 (1996) arXiv:hep-th/9604002.

[3] W. Siegel, Phys. Lett. B 84, 193 (1979).

[4] P. E. Haagensen, Mod. Phys. Lett. A 7, 893 (1992) arXiv:hep-th/9111015.

[5] D. E. Carneiro, A. P. Baeta Scarpelli, M. Sampaio and M. C. Nemes, JHEP 0312, 044 (2003) arXiv:hep-th/0309188.

[6] D. M. Capper, D. R. T. Jones and P. van Nieuwenhuizen, Nucl. Phys. B 167, 479 (1980).

[7] W. Beenakker, R. Hopker and P. M. Zerwas, Phys. Lett. B 378, 159 (1996) arXiv:hep-ph/9602378.

[8] W. Hollik, E. Kraus and D. Stockinger, Eur. Phys. J. C 11, 365 (1999) arXiv:hep-ph/9907393].

[9] W. Siegel, Phys. Lett. B 94, 37 (1980).

[10] D. Stockinger, JHEP 0503, $076(2005)$ arXiv:hep-ph/0503129].

[11] Y. L. Wu, Int. J. Mod. Phys. A 18, 5363 (2003) arXiv:hep-th/0209021.

[12] Y. L. Wu, Mod. Phys. Lett. A 19, 2191 (2004) arXiv:hep-th/0311082.

[13] Y. L. Ma and Y. L. Wu, Int. J. Mod. Phys. A 21, 6383 (2006) [arXiv:hep-ph/0509083].

[14] Y. L. Ma and Y. L. Wu, Phys. Lett. B 647, 427 (2007) arXiv:hep-ph/0611199.

[15] J. W. Cui and Y. L. Wu, Int. J. Mod. Phys. A 23, 2861 (2008) [arXiv:0801.2199 [hep-ph]].

[16] Y. B. Dai and Y. L. Wu, Eur. Phys. J. C 39, S1 (2005) arXiv:hep-ph/0304075.

[17] J. Wess and B. Zumino, Phys. Lett. B 49, 52 (1974).

[18] J. Iliopoulos and B. Zumino, Nucl. Phys. B 76, 310 (1974).

[19] P. K. Townsend and P. van Nieuwenhuizen, Phys. Rev. D 20, 1832 (1979).

[20] E. Sezgin, Nucl. Phys. B 162, 1 (1980).

[21] P. Majumdar, E. C. Poggio and H. J. Schnitzer, Phys. Rev. D 21, 2203 (1980).

[22] H. K. Dreiner, H. E. Haber, S. P. Martin Supersymmetry (CUP draft Sept. 2004)

[23] J. M. Jauch and F. Rohrlich, "The Theory of Photons and Electrons", Springer-Verlag, New York, Heidelberg, Berlin, 1976.

[24] A. Denner, H. Eck, O. Hahn and J. Kublbeck, Nucl. Phys. B 387, 467 (1992). 
[25] E. I. Gates and K. L. Kowalski, Phys. Rev. D 37, 938 (1988).

[26] J. Gluza and M. Zralek, Phys. Rev. D 45, 1693 (1992). 